\title{
ERROR ESTIMATES OF GAUSSIAN-TYPE QUADRATURE FORMULAE FOR ANALYTIC FUNCTIONS ON ELLIPSES-A SURVEY OF RECENT RESULTS*
}

\author{
D. LJ. DJUKIĆ $\dagger$, R. M. MUTAVDŽIĆ DJUKIĆ ${ }^{\dagger}$, A. V. PEJČEV ${ }^{\dagger}$, AND M. M. SPALEVIĆ $^{\dagger}$
}

Abstract. This paper presents a survey of recent results on error estimates of Gaussian-type quadrature formulas for analytic functions on confocal ellipses.

Key words. error estimate, remainder term, analytic function, contour integral representation

AMS subject classifications. 65D32, 65D30, 41A55

1. Error bounds of quadratures with simple and multiple nodes for analytic functions. Let $\Gamma$ be a simple closed curve in the complex plane encompassing the interval $[-1,1]$, and let $\mathcal{D}$ be its interior. Suppose $f$ is a function that is analytic in $\mathcal{D}$ and continuous on $\overline{\mathcal{D}}$. Taking any system of $m$ distinct points $\left\{\xi_{1}, \ldots, \xi_{m}\right\}$ in $\mathcal{D}$ and $m$ positive integers $n_{1}, \ldots, n_{m}$, the error in the Hermite interpolating polynomial of $f$ at a point $t \in \mathcal{D}$ can be expressed in the form (see, e.g., Mysovskih [56, Chapter 2], Gončarov [23, Chapter 5])

$$
r_{m}(f ; t)=f(t)-\sum_{\nu=1}^{m} \sum_{i=0}^{n_{\nu}-1} l_{\nu, i} f^{(i)}\left(\xi_{\nu}\right)=\frac{1}{2 \pi i} \oint_{\Gamma} \frac{f(z) \Omega_{m}(t)}{(z-t) \Omega_{m}(z)} d z,
$$

where $l_{\nu, i}(t)$ are the fundamental functions of Hermite interpolation and

$$
\Omega_{m}(z)=\prod_{\nu=1}^{m}\left(z-\xi_{\nu}\right)^{n_{\nu}} .
$$

By multiplying (1.1) by the weight function $w(t)$ and integrating with respect to $t$ over $(-1,1)$, we get a contour integral representation for the remainder term $R_{m}(f)$ in a quadrature formula with multiple nodes:

$$
R_{m}(f)=I(f ; w)-\sum_{\nu=1}^{m} \sum_{i=0}^{n_{\nu}-1} A_{i, \nu} f^{(i)}\left(\xi_{\nu}\right)=\frac{1}{2 \pi i} \oint_{\Gamma} K_{m}(z ; w) f(z) d z,
$$

where

$$
I(f ; w)=\int_{-1}^{1} f(t) w(t) d t, \quad A_{i, \nu}=\int_{-1}^{1} l_{\nu, i}(t) w(t) d t,
$$

and the kernel $K_{m}(z)=K_{m}(z ; w)$ is given by

$$
K_{m}(z ; w)=\frac{\varrho_{m}(z ; w)}{\Omega_{m}(z)}, \quad \varrho_{m}(z ; w)=\int_{-1}^{1} \frac{\Omega_{m}(t)}{z-t} w(t) d t, \quad z \in \mathbb{C} \backslash[-1,1] .
$$

The integral representation (1.2) leads directly to the error estimate

$$
\left|R_{m}(f)\right| \leq \frac{l(\Gamma)}{2 \pi}\left(\max _{z \in \Gamma}\left|K_{m}(z)\right|\right)\left(\max _{z \in \Gamma}|f(z)|\right),
$$

\footnotetext{
${ }^{*}$ Received November 27, 2019. Accepted March 18, 2020. Published online on April 30, 2020. Recommended by L. Reichel. Research supported in part by the Serbian Ministry of Education, Science and Technological Development. Contract no. 451-03-68 / 2020-14 / 200105.

$\dagger$ Department of Mathematics, University of Belgrade, Faculty of Mechanical Engineering, Kraljice Marije 16, 11120 Belgrade 35, Serbia(\{ddjukic, rmutavdzic, apejcev, mspalevic\}@mas.bg.ac.rs\}).
} 
where $l(\Gamma)$ is the length of the contour $\Gamma$. A general estimate can be obtained by Hölder's inequality. Namely,

$$
\left|R_{m}(f)\right|=\frac{1}{2 \pi}\left|\oint_{\Gamma} K_{m}(z) f(z) d z\right| \leq \frac{1}{2 \pi}\left(\oint_{\Gamma}\left|K_{m}(z)\right|^{p}|d z|\right)^{1 / p}\left(\oint_{\Gamma}|f(z)|^{q}|d z|\right)^{1 / q}
$$

i.e.,

$$
\left|R_{m}(f)\right| \leq \frac{1}{2 \pi}\left\|K_{m}\right\|_{p}\|f\|_{q},
$$

where $1 \leq p \leq+\infty, 1 / p+1 / q=1$, and

$$
\|f\|_{p}:= \begin{cases}\left(\oint_{\Gamma}|f(z)|^{p}|d z|\right)^{1 / p}, & 1 \leq p<+\infty \\ \max _{z \in \Gamma}|f(z)|, & p=+\infty .\end{cases}
$$

The case $(p, q)=(+\infty, 1)$ gives an $L^{\infty}$-error bound (1.3) when the $L^{1}$-norm of $f$ is estimated by its $L^{\infty}$-norm. On the other hand, the case $(p, q)=(1,+\infty)$ yields an $L^{1}$-error bound:

$$
\left|R_{m}(f)\right| \leq \frac{1}{2 \pi}\left(\oint_{\Gamma}\left|K_{m}(z)\right||d z|\right)\left(\max _{z \in \Gamma}|f(z)|\right)
$$

which is evidently stronger than (1.3) since

$$
\oint_{\Gamma}\left|K_{m}(z)\right||d z| \leq l(\Gamma)\left(\max _{z \in \Gamma}\left|K_{m}(z)\right|\right) .
$$

In order to obtain the estimates (1.3) and (1.4), one needs to study the magnitude of $\left|K_{m}(z)\right|$ on $\Gamma$ or the quantity

$$
L_{m}(\Gamma):=\frac{1}{2 \pi} \oint_{\Gamma}\left|K_{m}(z)\right||d z|,
$$

respectively.

A common choice for the contour $\Gamma$ is one of the confocal ellipses with foci at the points $\mp 1$ and the sum of semi-axes $\rho>1$,

$$
\mathcal{E}_{\rho}=\left\{z \in \mathbb{C}: z=\frac{1}{2}\left(\rho e^{i \theta}+\rho^{-1} e^{-i \theta}\right), 0 \leq \theta<2 \pi\right\} .
$$

For such $\Gamma$ we studied the estimates (1.3) (see [39, 49]) and (1.4) (see [42]) for the Gauss-Turán quadrature formulas $\left(n_{\nu}=2 s+1, \nu=1,2, \ldots, n ; s \in \mathbb{N}_{0}\right)$ when $w$ is one of the four generalized Chebyshev weight functions
(a) $w_{1}(t)=\left(1-t^{2}\right)^{-1 / 2}$,
(b) $w_{2}(t)=\left(1-t^{2}\right)^{1 / 2+s}$,
(c) $w_{3}(t)=(1-t)^{-1 / 2}(1+t)^{1 / 2+s}$,
(d) $w_{4}(t)=(1-t)^{1 / 2+s}(1+t)^{-1 / 2}$;

see $[5,39,70]$.

The error term in Gaussian quadratures $(s=0)$ for these weights was studied in the case $p=+\infty$ by Gautschi and Varga [21] (also see [20]) and in the case $p=1$ by Hunter [28]. Also see $[12,29,72,82,83,84]$ for this topic. 
Gautschi and Varga [21] (also see [20]) presented the original method for the error bound of the type (1.3),

$$
\left|R_{n}(f)\right| \leq \frac{l\left(\mathcal{E}_{\rho}\right)}{2 \pi}\left(\max _{z \in \mathcal{E}_{\rho}}\left|K_{n}(z)\right|\right)\left(\max _{z \in \mathcal{E}_{\rho}}|f(z)|\right),
$$

in Gaussian quadrature,

$$
\int_{-1}^{1} f(t) w(t) d t=\sum_{\nu=1}^{n} A_{\nu} f\left(\xi_{\nu}\right)+R_{n}(f)
$$

with respect to some of the four Chebyshev weight functions $w(t)$ (cf. (1.7) with $s=0$ ). They attempted to determine exactly where on the confocal ellipses $\mathcal{E}_{\rho}$ the kernel of the error functional attains its maximum modulus, succeeded in answering this question for the Chebyshev weight functions, and provided empirical results for more general Jacobi weights. In this survey we mention how other authors, including ourselves, contributed to this method.

Setting

$$
z=\frac{1}{2}\left(\xi+\xi^{-1}\right), \quad \xi=\rho e^{i \theta}, \quad \text { and } \quad|d z|=2^{-1 / 2} \sqrt{a_{2}-\cos 2 \theta} d \theta
$$

where we denote

$$
a_{j}=a_{j}(\rho)=\frac{1}{2}\left(\rho^{j}+\rho^{-j}\right), \quad j \in \mathbb{N}, \rho>1
$$

(1.5) reduces to

$$
L_{m}\left(\mathcal{E}_{\rho}\right):=\frac{1}{2 \pi \sqrt{2}} \int_{0}^{2 \pi} \frac{\left|\varrho_{m}(z ; w)\right|\left(a_{2}-\cos 2 \theta\right)^{1 / 2}}{\left|\Omega_{m}(z)\right|} d \theta .
$$

This integral can be evaluated numerically by using a quadrature formula. Moreover, for Gauss-Turán quadrature formulas with $w(t)$ being one of the four generalized Chebyshev weights (1.7), we obtained explicit expressions for $L_{m}\left(\mathcal{E}_{\rho}\right)$ or the bounds thereof; see Milovanović and Spalević [42]. In [42], for the first time, we considered error bounds for GaussTurán quadrature formulas based on expanding the remainder term into a series. These methods originated with Hunter [28], who applied them to the Gaussian quadrature formula (1.9) with $w(t)$ being one of the four Chebyshev weights (cf. (1.7) with $s=0$ ).

One of us started working on this topic some 20 years ago. Our first published paper on this topic was Milovanović and Spalević [39]. In the meanwhile, we and our collaborators published a number of related papers; see [9], [32]-[34], [39]-[46], [37], [49]-[55], [69], [73]-[81], [86]-[92]. We considered in our papers mainly error bounds and estimates of the type $L^{\infty}, L^{1}$, and the ones based on expanding the remainder term into a series for the quadrature formulas with multiple and simple nodes of Gaussian type, including Kronrod extensions, Radau, and Lobatto modifications, mainly with the specific weight functions such as the generalized and ordinary Chebyshev weights (see [5, 70]), the Gori-Micchelli weights (see [24]), the Bernstein-Szegó weight functions (see [19]), and some of their modifications; see [17]. Recently, we have been considering these problems for quadratures for Fourier coefficients in [6,31,36, 47].

For Gaussian quadrature formulas with multiple nodes, in particular Gauss-Turán quadrature formulas and their Kronrod extensions, numerically stable methods for their computation have been proposed (see $[15,18,48,85])$. For the theory of Gauss-Turán quadrature formulas, 
see the book [22]; see also the survey papers [35, 38]. A nice and detailed survey of Kronrod rules in the last fifty years is provided by Notaris [68]. In particular, Section 3.3 in [68] is dedicated to error bounds for analytic functions. Notaris's papers (see [57]-[68]) have had a significant influence on the development of our methods, especially lately, and especially the papers in which he considers the error estimates in quadratures for analytic functions on circles.

In two of our papers $[41,46]$ on the subject, we use circles instead of ellipses, and the results follow from the ones in [93].

Separately, following the ideas in [93], we derived some $L^{2}$-estimates for Gauss-Turán quadrature formulas in [46]. Some of our methods under consideration are also applied to quadrature rules with multiple nodes for evaluating integrals with strong singularities; see [44]. We present a computable upper bound of the error for Gauss-Turán quadrature formulas for analytic integrands in a neighborhood of the set of integration. This bound remains valid for arbitrary weight functions (cf. [89]), and the corresponding method is described in Section 5.

In Section 2 the error bounds for ordinary Gaussian quadrature formulas are discussed. Section 3 describes $L^{\infty}$-error bounds for Gauss-Radau rules, and in Section 4 for GaussKronrod quadrature formulas for the Bernstein-Szegó weight functions; cf. [80] and [9]. Additionally, we shall prove some statements which we use in those papers. In Section 6 we present the three different error estimates mentioned above for the Kronrod extensions of generalizations of the Micchelli-Rivlin quadrature formula for the Fourier-Chebyshev coefficients with the highest algebraic degree of precision; cf. [54]. Finally, in Section 7 we mention, only briefly, error estimates for analytic functions on concentric circles.

2. Error bounds for Gaussian quadrature formulas. For a Gaussian quadrature formula (1.9) relative to a nonnegative weight function $w(t)$, it holds that $R_{n}(f)=0$ for all $f \in \mathbb{P}_{2 n-1}$ and the node polynomial $\pi_{n}(t)$ is the $n$th degree (monic) orthogonal polynomial with respect to the weight function $w(t)$. Further,

$$
\varrho_{n}(z ; w)=\int_{-1}^{1} \frac{\pi_{n}(t)}{z-t} w(t) d t,
$$

and the kernel $K_{n}(z)=\varrho_{n}(z ; w) / \pi_{n}(z)$ can be computed using a basic three-term recurrence relation, which is satisfied by the corresponding orthogonal polynomials $\pi_{n}$; for more details see [21]. In [21] it is shown that it is possible to find explicit formulas for the kernels of the four Chebyshev weights (cf. (1.7) with $s=0$ ).

We discuss in some detail the case of the first Chebyshev weight $w=w_{1}$. Here one finds [21, Subsection 5.1], for $z \in \mathcal{E}_{\rho}$, the explicit formula

$$
\left|K_{n}\left(z ; w_{1}\right)\right|=\frac{2 \pi}{\rho^{n}}\left[a_{2}(\rho)-\cos 2 \theta\right]^{-1 / 2}\left[a_{2 n}(\rho)+\cos 2 n \theta\right]^{-1 / 2}, \quad 0 \leq \theta \leq 2 \pi,
$$

where the $a_{j}=a_{j}(\rho)$ given by (1.10) appear in all the formulae for the kernels belonging to Chebyshev weight functions. In particular, they satisfy the following inequality (cf. [16, Subsection 3.1]).

LEMMA 2.1. For any $\rho>1$, it holds that

$$
\frac{a_{2 n}(\rho)-1}{a_{2}(\rho)-1} \geq n^{2}, \quad n=1,2, \ldots .
$$

This is the key for proving that $\left|K_{n}\left(z ; w_{1}\right)\right|$ attains its maximum on the real axis on $\mathcal{E}_{\rho}$ :

THEOREM 2.1. For any $\rho>1$ and each $n \in \mathbb{N}$, one has

$$
\max _{z \in \mathcal{E}_{\rho}}\left|K_{n}\left(z ; w_{1}\right)\right|=K_{n}\left(\frac{1}{2}\left(\rho+\rho^{-1}\right) ; w_{1}\right)=\frac{4 \pi}{\rho^{n}} \frac{1}{\left(\rho-\rho^{-1}\right)\left(\rho^{n}+\rho^{-n}\right)} .
$$


Proof. According to (2.1) one must prove that

$$
\left(a_{2}-\cos 2 \theta\right)\left(a_{2 n}+\cos 2 n \theta\right) \geq\left(a_{2}-1\right)\left(a_{2 n}+1\right), \quad 0 \leq \theta \leq \pi / 2,
$$

where $a_{j}=a_{j}(\rho)$ (there is symmetry with respect to both coordinate axes). Simple trigonometry will show that (2.3), for $\theta>0$, is equivalent to

$$
a_{2 n}+1-\left(a_{2}-1\right) \frac{\sin ^{2} n \theta}{\sin ^{2} \theta}-2 \sin ^{2} n \theta \geq 0 .
$$

Since $|\sin n \theta / \sin \theta| \leq n$, the left-hand side of (2.4) is greater than

$$
a_{2 n}+1-n^{2}\left(a_{2}-1\right)-2=\left(a_{2}-1\right)\left(\frac{a_{2 n}-1}{a_{2}-1}-n^{2}\right),
$$

which is nonnegative by Lemma 2.1 .

For the Chebyshev weight of the second kind, $w(t)=w_{2}(t)$ (with $s=0$ in (1.7)), one finds for $z \in \mathcal{E}_{\rho}$ that

$$
\left|K_{n}\left(z ; w_{2}\right)\right|=\frac{\pi}{\rho^{n+1}}\left[a_{2}(\rho)-\cos 2 \theta\right]^{1 / 2}\left[a_{2 n+2}(\rho)-\cos 2(n+1) \theta\right]^{-1 / 2}, \quad 0 \leq \theta \leq 2 \pi .
$$

From this it is rather straightforward to show that the maximum of $\left|K_{n}\left(\cdot ; w_{2}\right)\right|$ on $\mathcal{E}_{\rho}$ is attained on the imaginary axis, i.e., for $\theta=\pi / 2$, if $n$ is odd. If $n$ is even, however, then this is true only for $\rho$ sufficiently large. The precise result is as follows [20]:

THEOREM 2.2. There holds

$$
\max _{z \in \mathcal{E}_{\rho}}\left|K_{n}\left(z ; w_{2}\right)\right|=K_{n}\left(\frac{i}{2}\left(\rho-\rho^{-1}\right) ; w_{2}\right)=\frac{\pi}{\rho^{n+1}} \frac{\rho+\rho^{-1}}{\rho^{n+1}+(-1)^{n} \rho^{-(n+1)}}
$$

for all $\rho>1$ if $n$ is odd and for all $\rho>\rho_{n+1}$ if $n$ is even, where $\rho_{n+1}$ is the unique root of

$$
\frac{\rho+\rho^{-1}}{\rho^{n+1}+\rho^{-(n+1)}}=\frac{1}{n+1}, \quad \rho>1 .
$$

The following numerical values of $\rho_{n+1}$ in Table 2.1 show that the ellipse $\mathcal{E}_{\rho}$ has to be quite slim for (2.5) to fail when $n$ is even. Indeed, it can be shown that $\varrho_{n}$ decreases to 1 as $n \rightarrow \infty$.

\begin{tabular}{c|ccccc}
\multicolumn{7}{c}{ TABLE 2.1} \\
The roots $\rho_{n+1}$. \\
$n$ & 2 & 4 & 8 & 16 & 32 \\
\hline$\rho_{n+1}$ & 1.932 & 1.618 & 1.386 & 1.232 & 1.136
\end{tabular}

Finally, for the Chebyshev weight of the third kind, $w(t)=w_{3}(t)$ (with $s=0$ in (1.7)), an argument similar to the one in the proof of Theorem 2.1 will establish:

THEOREM 2.3. For any $\rho>1$ and each $n \in \mathbb{N}$, there holds

$$
\max _{z \in \mathcal{E}_{\rho}}\left|K_{n}\left(z ; w_{3}\right)\right|=K_{n}\left(\frac{1}{2}\left(\rho+\rho^{-1}\right) ; w_{3}\right)=\frac{2 \pi}{\rho^{n+1 / 2}} \frac{\rho+1}{\rho-1} \frac{1}{\rho^{n+1 / 2}+\rho^{-n-1 / 2}} .
$$

The results for the Chebyshev weight of the fourth kind, $w(t)=w_{4}(t)$ (with $s=0$ in (1.7)), are easily deducible from those for $w_{3}(t)$ (with $s=0$ in (1.7)), as $w_{4}(t)=w_{3}(-t)$. 
The derivation of adequate bounds for $\left|R_{n}(f)\right|$ based on (1.8) is possible only if good estimates for $\max _{z \in \mathcal{E}_{\rho}}\left|K_{n}(z)\right|$ are available, especially if we know the location of the extremal point $\eta \in \mathcal{E}_{\rho}$ at which $\left|K_{n}\right|$ attains its maximum. In such a case, instead of looking for upper bounds for $\max _{z \in \mathcal{E}_{\rho}}\left|K_{n}(z)\right|$, one can simply try to calculate $\left|K_{n}(\eta, w)\right|$. In general, this may not be an easy task, but in the case of the Gauss-type quadrature formula (1.9), there exist effective algorithms for the calculation of $K_{n}(z)$ at any point $z$ outside $[-1,1]$; see [21].

The approach by Gautschi and Varga [21] (also see [20]) has been applied by Spalević in [86] to anti-Gaussian quadrature formulas (introduced by Laurie [30]) with Chebyshev weight functions (cf. (1.7) with $s=0$ ).

The same approach has been extended by Schira to symmetric weight functions under a monotonicity requirement (either $w(t) \sqrt{1-t^{2}}$ is increasing on $(0,1)$ or $w(t) / \sqrt{1-t^{2}}$ is decreasing on $(0,1)$ ), including certain Gegenbauer weight functions (see [84]). A generalization to the Lobatto case has been carried out by Hunter and Nikolov; see [29].

Schira proved [84, Theorem 3.2(b) on p. 302] that the kernel $K_{n}$ of a Gaussian quadrature rule with respect to a symmetric weight function $w$ on $(-1,1)$ satisfies the following condition: If $w(t) / \sqrt{1-t^{2}}$ is decreasing on $(0,1)$, then

$$
\max _{z \in \mathcal{E}_{\rho}}\left|K_{n}(z)\right|=\left|K_{n}\left(\frac{i}{2}\left(\rho-\rho^{-1}\right)\right)\right| \quad \text { for } \quad \rho \geq \rho_{n}^{*},
$$

where $\rho_{n}^{*}:=1+\sqrt{2}$ if $n \geq 1$ is odd, and if $n \geq 2$ is even, then $\rho_{n}^{*}$ is the greatest zero of

$$
d_{n}(\rho):=\left(\rho-\rho^{-1}\right)^{2}-4-\left(\rho^{2}-\rho^{-2}\right)^{2}\left(\frac{(n+1)^{2}}{\left(\rho^{n+1}-\rho^{-n-1}\right)^{2}}+\frac{(n+3)^{2}}{\left(\rho^{n+3}-\rho^{-n-3}\right)^{2}}\right) .
$$

The displayed values for $n$ even in [84, Table 1 on p. 302] suggest that $\rho_{n}^{*}$ rapidly converges to $1+\sqrt{2}(+0)$ as $n$ increases.

In a number of our papers, $w$ represents the classes of Bernstein-Szegő weight functions

$$
w(t) \equiv w_{\alpha, \beta, \delta}^{(\mp 1 / 2)}(t)=\frac{\left(1-t^{2}\right)^{\mp 1 / 2}}{\beta(\beta-2 \alpha) t^{2}+2 \delta(\beta-\alpha) t+\alpha^{2}+\delta^{2}}, \quad t \in(-1,1),
$$

or

$$
w(t) \equiv w_{\alpha, \beta, \delta}^{(\mp 1 / 2, \pm 1 / 2)}(t)=\frac{(1-t)^{\mp 1 / 2}(1+t)^{ \pm 1 / 2}}{\beta(\beta-2 \alpha) t^{2}+2 \delta(\beta-\alpha) t+\alpha^{2}+\delta^{2}}, \quad t \in(-1,1),
$$

where $0<\alpha<\beta, \beta \neq 2 \alpha,|\delta|<\beta-\alpha$, whose denominator is an arbitrary polynomial of exact degree 2 that remains positive on $[-1,1]$. The weight functions (2.6) and (2.7) have been studied extensively in [19].

For $\alpha=1, \beta=2 /(1+\gamma), \delta=0$, the weight functions (2.6) reduce to

$$
w(t) \equiv w_{\gamma}^{(\mp 1 / 2)}(t)=\frac{\left(1-t^{2}\right)^{\mp 1 / 2}}{1-\frac{4 \gamma}{(1+\gamma)^{2}} t^{2}}, \quad t \in(-1,1), \quad \gamma \in(-1,0],
$$

and the weight functions (2.7) to

$$
w(t) \equiv w_{\gamma}^{(\mp 1 / 2, \pm 1 / 2)}(t)=\frac{(1-t)^{\mp 1 / 2}(1+t)^{ \pm 1 / 2}}{1-\frac{4 \gamma}{(1+\gamma)^{2}} t^{2}}, \quad t \in(-1,1), \quad \gamma \in(-1,0] .
$$

Clearly, the four Chebyshev weights are special cases of (2.8), (2.9) with $\gamma=0$.

In [90], in the case of the weight function $w_{\gamma}^{(1 / 2)}(t)$ (cf. (2.8)), sufficient conditions were found to ensure the existence of a $\rho^{*}=\rho_{n}^{*}\left(=\rho^{*}(n, \gamma)\right)$ such that for each $\rho \geq \rho_{n}^{*}$, the kernel 
attains its maximum absolute value at the intersection points of the ellipse with the imaginary axis. For this special case, much smaller values for $\rho=\rho_{n}^{*}$ than the ones obtained by Schira (except for $\gamma$ close to 0 and $n$ even) were obtained, especially for large values of $n$. In [77], the case of the more general class of weight functions $w_{\alpha, \beta, \delta}^{(1 / 2)}(t)$ (cf. (2.6)), sufficient conditions are found to ensure the existence of a $\rho^{*}=\rho_{n}^{*}\left(=\rho^{*}(n, \alpha, \beta, \delta)\right)$ such that for each $\rho \geq \rho_{n}^{*}$, the kernel attains its maximum absolute value at the intersection points of the ellipse with either the real or the imaginary axis. This leads to effective error bounds of the corresponding Gauss quadratures. The approach we use in this paper, which differs from the one in [90], ensures that the values $\rho^{*}=\rho_{n}^{*}\left(=\rho^{*}(n, \alpha, \beta, \delta)\right)$ are estimated very precisely. The results obtained in [84] cannot be used here in the general case $(\delta \neq 0)$, since the weight function under consideration is not symmetric. Analogous investigations have been carried out for the other weight functions of Bernstein-Szegô type (2.8), (2.9), and (2.6), (2.7), in [92], [87], and [73], [74], respectively. A survey of these methods for all the weight functions (2.8), (2.9) can be found in [88].

In [69] Gaussian quadrature formulae corresponding to some modifications of the four Chebyshev weights $w_{j}(t), j=1,2,3,4$, (cf. (1.7) with $s=0$ ) are considered, i.e., corresponding to the weights

$$
\hat{w}_{j}(t)=\left[\pi_{n}^{(j)}(t)\right]^{2} w_{j}(t), \quad j=1,2,3,4,
$$

discussed by Gautschi and Li in [17]. Here $\pi_{n}^{(j)}(t)$ is the corresponding (monic) orthogonal polynomial relative to $w_{j}$. In the case of analytic integrands, the error of these quadrature formulas is represented as a contour integral with a complex kernel. We studied the kernels, on elliptic contours, of the mentioned quadrature formulas and derived effective $L^{\infty}, L^{1}$-error bounds for them as well as error bounds based on expanding the error into a series. In addition, we analysed in a rather simple manner the behavior of the modulus of the corresponding kernels on those ellipses in some cases by [69, Lemma 2.1].

In [51] the remainder term of a Gaussian quadrature formula and its Kronrod extension for analytic functions is represented as a contour integral with a complex kernel. We studied these kernels on elliptic contours for the Chebyshev weight functions of the first, second, and third kinds (cf. (1.7) with $s=0$ ) and derived representations of their differences. Using these representations and following Kronrod's method of obtaining a practical error estimate in numerical integration (see e.g., [68]), we obtained new error estimates for Gaussian quadratures.

3. Error bounds for Gauss-Radau and Gauss-Lobatto quadrature formulas. In [12] Gautschi considered Gauss-Radau quadrature rules

$$
\int_{-1}^{1} f(t) w(t) d t=\sum_{\nu=1}^{n} \lambda_{\nu} f\left(\tau_{\nu}\right)+\lambda_{n+1} f(c)+R_{n}(f)
$$

where $c=-1$, or $c=1$, and Gauss-Lobatto quadrature rules

$$
\int_{-1}^{1} f(t) w(t) d t=\lambda_{0} f(-1)+\sum_{\nu=1}^{n} \lambda_{\nu} f\left(\tau_{\nu}\right)+\lambda_{n+1} f(1)+R_{n}(f)
$$

with respect to the four Chebyshev weight functions $w_{j}, j=1,2,3,4$, (cf. (1.7) with $s=0$ ) and derived explicit expressions for the corresponding kernels $K\left(z ; w_{j}\right)$ in terms of the variable $u=\rho e^{i \theta}$. For Gauss-Lobatto quadratures it is proved that $\left|K_{n+2}\left(z ; w_{1}\right)\right|$ attains its maximum on $\mathcal{E}_{\rho}$ on the real axis (cf. [12, Theorem 4.1]). For $w_{2}, w_{3}$, and $w_{4}$, empirical results 
and conjectures on the location of the maximum were presented. These conjectures have been proved later by Schira in [83].

For Gauss-Radau quadratures with a fixed node at -1 , Gautschi proved that the corresponding kernels $K_{n+1}(z ; w)$ for the Chebyshev weight functions $w(t)=w_{1}(t)$ and $w(t)=w_{4}(t)$ attains their maximum modulus on $\mathcal{E}_{\rho}$ on the negative real axis (cf. [12, Theorems 4.4 and 4.5]). Milovanović, Spalević, and Pranić [50] proved and confirmed the corresponding empirical results from [12] in the case $w=w_{2}$, and Pejčev and Spalević [76] proved and confirmed the corresponding empirical results from [12] in the cases $w=w_{3}$ and $w=w_{4}$.

For Gauss-Radau and Gauss-Lobatto quadrature formulae with multiple end points with a Chebyshev weight function (see (1.7), with $s=0$ ) and for integrands analytic on ellipses, the explicit expression for the corresponding kernels are derived by Gautschi and $\mathrm{Li}$ in [16]. We determined the locations on the ellipses where the maximum modulus of the kernel is attained. The obtained values confirmed in part the corresponding conjectured values given by Gautschi and $\mathrm{Li}$ [16]. We have performed for those cases a more detailed numerical study. The effective error bounds for those quadrature formulas are derived in [32, 33, 75].

Without loss of generality, we consider the Gauss-Radau quadrature formula (3.1) for the (nonnegative) weight function $w$ on the interval $[-1,1]$ and an additional node $c=-1$, where $\tau_{\nu}$ are the zeros of the $n$th degree (monic) orthogonal polynomial $\pi_{n}^{R}(\cdot)=\pi_{n}^{R}\left(\cdot ; w^{R}\right)$ relative to the weight function $w^{R}(t)=(1+t) w(t)$. It is well known that formula (3.1) has all weights positive and precise degree of exactness $d=2 n$, i.e., $R_{n}(f)=0$ for all $f \in \mathcal{P}_{2 n}$, where $\mathcal{P}_{2 n}$ represents the set of all polynomials of degree less than or equal to $2 n$.

In the paper [80], $w$ is one of the four weight functions of Bernstein-Szegó type (2.8) or (2.9). For analytic functions, the remainder term of this quadrature formula can be represented as a contour integral with a complex kernel as in (1.2). We study the kernel on elliptic contours (1.6) for the given quadrature formula. Starting from the explicit expression of the kernel, we determine the locations on the ellipses where the maximum modulus of the kernel is attained. So we derive effective error bounds of the form (1.3) for this quadrature formula. An alternative approach, which initiated this research, has been proposed by S. Notaris [65]. The kernel is given by

$$
K_{n}(z) \equiv K_{n}^{R}(z ; w)=\frac{\varrho_{n}^{R}(z)}{(1+z) \pi_{n}^{R}(z)}, \quad z \notin[-1,1],
$$

where

$$
\varrho_{n}(z) \equiv \varrho_{n, w}^{R}(z)=\int_{-1}^{1} \frac{\pi_{n}^{R}(t)}{z-t} w^{R}(t) d t .
$$

In the case of $w=w_{\gamma}^{(-1 / 2)}$, we have (cf. [65, p. 4])

$$
\varrho_{n}^{(-1 / 2)}(z)=\int_{-1}^{1} \frac{\pi_{n}^{(-1 / 2,1 / 2)}(t)}{z-t} w_{\gamma}^{(-1 / 2,1 / 2)}(t) d t
$$

and

$$
K_{n}^{(-1 / 2)}(z)=\frac{\varrho_{n}^{(-1 / 2)}(z)}{(1+z) \pi_{n}^{(-1 / 2,1 / 2)}(z)}
$$

where

$$
\pi_{1}^{(-1 / 2,1 / 2)}(z)=z-\frac{1+\gamma}{2}, \quad \pi_{n}^{(-1 / 2,1 / 2)}(z)=\frac{1}{2^{n}}\left[V_{n}(z)-\gamma V_{n-2}(z)\right] \quad(n \geq 2),
$$


and $V_{n}(\cos \theta)=\cos ((n+1 / 2) \theta) / \cos (\theta / 2)$ is the Chebyshev polynomial of the third kind. Referring to equation (2.3) from [87], upon division by $1+z$ with $z=\left(\xi+\xi^{-1}\right) / 2$, we directly get the explicit expression for $K_{n}^{(-1 / 2)}(z)$.

In the case of $w=w_{\gamma}^{(1 / 2)}$, we have

$$
\varrho_{n}^{(1 / 2)}(z)=\int_{-1}^{1} \frac{(1+t) \pi_{n}^{(1 / 2)^{R}}(t)}{z-t} w_{\gamma}^{(1 / 2)}(t) d t .
$$

We know (see e.g., [65, pp. 7-8]) that

$$
(1+t) \pi_{n}^{(1 / 2)^{R}}(t)=\pi_{n+1}^{(1 / 2)}(t)-\frac{\pi_{n+1}^{(1 / 2)}(-1)}{\pi_{n}^{(1 / 2)}(-1)} \pi_{n}^{(1 / 2)}(t), \quad n \in \mathbb{N},
$$

where

$$
\pi_{n}^{(1 / 2)}(-1)=\frac{(-1)^{n}}{2^{n}}[n+1-\gamma(n-1)], \quad n \in \mathbb{N},
$$

and

$$
\pi_{n}^{(1 / 2)}(t)=\frac{1}{2^{n}}\left[U_{n}(t)-\gamma U_{n-2}(t)\right], \quad n \in \mathbb{N} .
$$

Here $U_{n}(\cos \theta)=\sin ((n+1) \theta) / \sin \theta$ is the Chebyshev polynomial of the second kind. For the formulas for the polynomials $\pi_{n}^{(-1 / 2,1 / 2)}, \pi_{1}^{(-1 / 2,1 / 2)}$, and $\pi_{n}^{(1 / 2)}$; see [19, Eqs. (3.10), $(3.10)^{1}$, (3.9), with $\alpha=1, \beta=2 /(1+\gamma)$ and $\left.\delta=0\right]$. Therefore for $n \in \mathbb{N}$, we have

$$
\begin{aligned}
(1+t) \pi_{n}^{(1 / 2)^{R}}(t) & =\pi_{n+1}^{(1 / 2)}(t)+\frac{1}{2} \cdot \frac{n+2-n \gamma}{n+1-(n-1) \gamma} \pi_{n}^{(1 / 2)}(t) \\
& =\frac{1}{2^{n+1}}\left[U_{n+1}(t)-\gamma U_{n-1}(t)+\frac{n+2-n \gamma}{n+1-(n-1) \gamma}\left(U_{n}(t)-\gamma U_{n-2}(t)\right)\right] .
\end{aligned}
$$

Then the explicit expression for $K_{n}^{(1 / 2)}(z)$ follows by the results from [90] and the well-known fact that (see e.g., [21, p. 8])

$$
\int_{-1}^{1} \frac{U_{n}(t)}{z-t} \sqrt{1-t^{2}} d t=\int_{0}^{\pi} \frac{\sin (n+1) \theta \sin \theta}{z-\cos \theta} d \theta=\frac{\pi}{\xi^{n+1}}, \quad z=\frac{1}{2}\left(\xi+\xi^{-1}\right) .
$$

Similarly, in the case of $w=w_{\gamma}^{(-1 / 2,1 / 2)}$, we have

$$
\varrho_{n}^{(-1 / 2,1 / 2)}(z)=\int_{-1}^{1} \frac{(1+t) \pi_{n}^{(-1 / 2,1 / 2)^{R}}(t)}{z-t} w_{\gamma}^{(-1 / 2,1 / 2)}(t) d t,
$$

where (see [65, p. 13])

$$
(1+t) \pi_{n}^{(-1 / 2,1 / 2)^{R}}(t)=\pi_{n+1}^{(-1 / 2,1 / 2)}(t)+\frac{1}{2} \cdot \frac{2 n+3-(2 n-1) \gamma}{2 n+1-(2 n-3) \gamma} \pi_{n}^{(-1 / 2,1 / 2)}(t) .
$$

Then the explicit expression for $K_{n}^{(-1 / 2,1 / 2)}(z)$ follows by results from [87] and the wellknown fact that (see e.g., [21, p. 9])

$$
\int_{-1}^{1} \frac{V_{n}(t)}{z-t} \sqrt{\frac{1+t}{1-t}} d t=\int_{0}^{\pi} \frac{2 \cos \left(n+\frac{1}{2}\right) \theta \cos \frac{\theta}{2}}{z-\cos \theta} d \theta=\frac{2 \pi(\xi+1)}{\left(\xi-\xi^{-1}\right) \xi^{n+1}}, \quad z=\frac{1}{2}\left(\xi+\xi^{-1}\right) .
$$



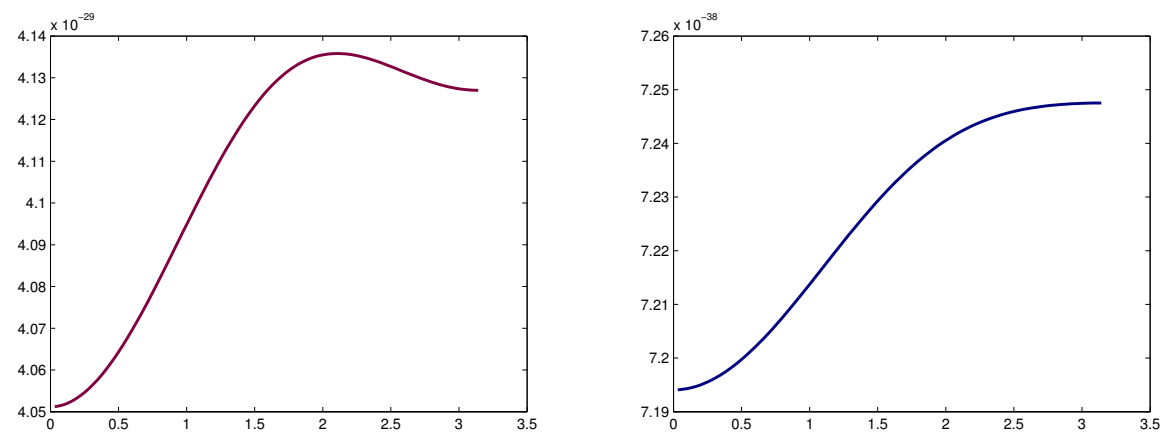

FIG. 3.1. The graphs of the modulus of the kernel $\left|K_{n}^{(1 / 2)}(z)\right|$ for $\gamma=-0.5, n=10$ and $\rho=20$ (left) and $\rho=50$ (right), respectively.

Finally, in the case of $w=w_{\gamma}^{(1 / 2,-1 / 2)}$, we have

$$
(1+t) w_{\gamma}^{(1 / 2,-1 / 2)}(t)=w_{\gamma}^{(1 / 2)}(t), \quad t \in[-1,1]
$$

so the explicit expression for $K_{n}^{(1 / 2,-1 / 2)}(z)$ follows by equations (2.2) and (2.4) from [90].

REMARK 3.1. Clearly, by substituting $t:=-t$, the quadrature formula

$$
\begin{aligned}
\int_{-1}^{1} f(t) w_{\gamma}^{(-1 / 2,1 / 2)}(t) d t=\sum_{\nu=1}^{n} & \lambda_{\nu}^{(-1 / 2,1 / 2)} f\left(\tau_{\nu}^{(-1 / 2,1 / 2)}\right) \\
& +\lambda_{n+1}^{(-1 / 2,1 / 2)} f(1)+R_{n}^{(-1 / 2,1 / 2)}(f)
\end{aligned}
$$

with the fixed node -1 (instead of 1 ) reduces to the quadrature formula

$$
\begin{aligned}
\int_{-1}^{1} f(t) w_{\gamma}^{(1 / 2,-1 / 2)}(t) d t=\sum_{\nu=1}^{n} & \lambda_{\nu}^{(1 / 2,-1 / 2)} f\left(\tau_{\nu}^{(1 / 2,-1 / 2)}\right) \\
& +\lambda_{n+1}^{(1 / 2,-1 / 2)} f(-1)+R_{n}^{(1 / 2,-1 / 2)}(f),
\end{aligned}
$$

and vice versa, the quadrature formula (3.4) with the fixed node +1 (instead of -1 ) reduces to the quadrature formula (3.3).

It turns out that, assuming that $\rho$ is large enough, say $\rho>\rho^{*}=\rho^{*}(n, \gamma)$, the moduli of all the four kernels attain their maxima on a coordinate axis. This observation is formalized by the remark at the end of this section. The smallest such $\rho^{*}$ tends to be close to 1 . An exception is the case of $w=w_{\gamma}^{(1 / 2)}$, when $\rho^{*}$ can become greater than 50 . The graphs of the modulus of the corresponding kernel $\left|K_{10}^{(1 / 2)}\right|$ for some values of $\rho$ are displayed in Figure 3.1.

Consider the integral

$$
I(f)=\int_{-1}^{1} f(t) w(t) d t
$$

with the four Chebyshev weights and the integrand $f(t)=f_{1}(t)=\frac{t^{4}}{\omega^{2}-t^{2}}$ (for some $\omega>1$ ) from [65]. Numerical experiments showed that, although the ellipse becomes almost circlelike for the corresponding $\rho^{*}$, the estimate can still be satisfactory as long as the integrand is analytic somewhere beyond $\rho^{*}$. Since

$$
\max _{z \in \mathcal{E}_{\rho}}\left|\frac{z^{4}}{\omega^{2}-z^{2}}\right|=\frac{a_{1}^{4}}{\omega^{2}-a_{1}^{2}},
$$




\section{ETNA}

Kent State University and

Johann Radon Institute (RICAM)

the maximum of the modulus of this function is attained at $\theta=0$. The obtained estimate is compared with the corresponding analogous estimates in equations (4.1) and (4.2), respectively, derived in [65], which are denoted by the superscript (Not), and the actual error bounds, which are denoted by Error; see the Tables 3.1 and 3.2.

TABLE 3.1

The values of $\hat{r}_{1}^{(N o t)}\left(f_{1}\right), \hat{r}_{2}^{(N o t)}\left(f_{1}\right), r\left(f_{1}\right)$, and Error for some $n, \gamma \in(-1,0)$, and $\omega$ in the case of the weight function $w_{\gamma}^{(1 / 2)}$.

\begin{tabular}{c|c|c|c|c}
$n, \gamma, \omega$ & $\hat{r}_{1}^{(\mathrm{Not})}\left(f_{1}\right)$ & $\hat{r}_{2}^{(\mathrm{Not})}\left(f_{1}\right)$ & $r\left(f_{1}\right)$ & Error \\
\hline $2,-0.91,6$ & $1.54(-6)$ & $8.68(-6)$ & $1.19(-5)$ & $1.95(-7)$ \\
$3,-0.7,8.5$ & $1.38(-8)$ & $1.90(-7)$ & $2.15(-7)$ & $1.10(-9)$ \\
$5,-0.5,12$ & $1.54(-14)$ & $4.98(-13)$ & $5.30(-13)$ & $6.49(-16)$ \\
$5,-0.3,8$ & $1.75(-12)$ & $3.88(-12)$ & $4.41(-11)$ & $7.25(-14)$ \\
$10,-0.1,15$ & $1.11(-29)$ & $7.55(-28)$ & $7.87(-28)$ & $1.81(-31)$ \\
$3,-0.1,3$ & $6.42(-5)$ & $4.37(-4)$ & $9.46(-4)$ & $5.31(-6)$ \\
$12,-0.05,15$ & $1.64(-35)$ & $1.26(-33)$ & $1.31(-33)$ & $2.08(-37)$
\end{tabular}

TABLE 3.2

The values of $\hat{r}_{1}^{(N o t)}\left(f_{1}\right), \hat{r}_{2}^{(N o t)}\left(f_{1}\right), r\left(f_{1}\right)$, and Error for some $n, \gamma \in(-1,0)$, and $\omega$ in the case of the weight function $w_{\gamma}^{(-1 / 2,1 / 2)}$.

\begin{tabular}{c|c|c|c|c}
$n, \gamma, \omega$ & $\hat{r}_{1}^{(\mathrm{Not})}\left(f_{1}\right)$ & $\hat{r}_{2}^{(\mathrm{Not})}\left(f_{1}\right)$ & $r\left(f_{1}\right)$ & Error \\
\hline $10,-0.89,3$ & $4.26(-17)$ & $1.13(-15)$ & $9.48(-16)$ & $9.86(-18)$ \\
$8,-0.75,5$ & $7.15(-17)$ & $1.55(-15)$ & $1.39(-15)$ & $1.18(-17)$ \\
$30,-0.6,8$ & $1.59(-72)$ & $1.34(-70)$ & $1.26(-70)$ & $1.82(-73)$ \\
$15,-0.5,6$ & $1.45(-32)$ & $6.00(-31)$ & $5.50(-31)$ & $2.08(-33)$ \\
$4,-0.3,10$ & $1.95(-10)$ & $3.46(-9)$ & $3.24(-9)$ & $3.00(-11)$ \\
$10,-0.1,11$ & $1.42(-26)$ & $6.11(-25)$ & $7.11(-25)$ & $1.89(-27)$
\end{tabular}

Analogous results are obtained for the Gauss-Lobatto quadrature formula (3.2) with one of the four weight functions of Bernstein-Szegó type (2.8) and (2.9); see [55]. An alternative approach, which has initiated this research, has been proposed by S. Notaris [59].

4. Error bounds for Gauss-Kronrod quadrature formulas. Let us consider in this section the Gauss-Kronrod quadrature formula for the (nonnegative) weight function $w$ on the interval $[-1,1]$ :

$$
\int_{-1}^{1} f(t) w(t) d t=Q_{n}[f]+R_{n}(f), \quad Q_{n}[f]=\sum_{\nu=1}^{n} \sigma_{\nu} f\left(\tau_{\nu}\right)+\sum_{\mu=1}^{n+1} \sigma_{\mu}^{*} f\left(\tau_{\mu}^{*}\right)
$$

where $\tau_{\nu}$ are the zeros of the $n$-th degree (monic) orthogonal polynomial $\pi_{n}(\cdot)$ relative to the weight function $w$, i.e., $\tau_{\nu}$ are the nodes of the corresponding $n$-point Gaussian-type quadrature formula $G_{n}$ relative to the weight function $w$,

$$
G_{n}[f]=\sum_{\nu=1}^{n} \lambda_{\nu} f\left(\tau_{\nu}\right)
$$

which has the algebraic degree of precision $2 n-1$. The nodes $\tau_{\mu}^{*}$ and all weights $\sigma_{\nu}, \sigma_{\mu}^{*}$ are chosen such that formula (4.1) has maximum degree of precision at least $3 n+1$, i.e., 
$R_{n}(f)=0$ for all $f \in \mathcal{P}_{3 n+1}$. It is known that the $\tau_{\mu}^{*}$ are zeros of a (monic) polynomial $\pi_{n+1}^{*}(\cdot ; w)$ of degree $n+1$ called the Stieltjes polynomial, which satisfies the orthogonality condition

$$
\int_{-1}^{1} \pi_{n+1}^{*}(t) \pi_{n}(t) t^{k} w(t) d t=0, \quad k=0,1, \ldots, n .
$$

In the paper [9], which has been initiated by the alternative approach proposed by S. Notaris [61], the weight function is again one of the four Bernstein-Szegó weight functions (2.8), (2.9). Let the corresponding (monic) orthogonal polynomials and Stieltjes polynomials relative to the weight functions (2.8), (2.9) be denoted by

$$
\begin{aligned}
\pi_{n}^{(\mp 1 / 2)}(\cdot) & =\pi_{n}\left(\cdot ; w^{(\mp 1 / 2)}\right), & \pi_{n}^{(\mp 1 / 2, \pm 1 / 2)}(\cdot) & =\pi_{n}\left(\cdot ; w^{(\mp 1 / 2, \pm 1 / 2)}\right), \\
\pi_{n+1}^{*(\mp 1 / 2)}(\cdot) & =\pi_{n+1}^{*}\left(\cdot ; w^{(\mp 1 / 2)}\right), & \pi_{n+1}^{*(\mp 1 / 2, \pm 1 / 2)}(\cdot) & =\pi_{n+1}^{*}\left(\cdot ; w^{(\mp 1 / 2, \pm 1 / 2)}\right),
\end{aligned}
$$

respectively. The kernel is given by

$$
K_{n}(z) \equiv K_{n}^{G K}(z ; w)=\frac{\varrho_{n}(z)}{\pi_{n}(z) \pi_{n+1}^{*}(z)}, \quad z \notin[-1,1]
$$

where

$$
\varrho_{n}(z) \equiv \varrho_{n, w}^{G K}(z)=\int_{-1}^{1} \frac{\pi_{n}(t) \pi_{n+1}^{*}(t)}{z-t} w(t) d t .
$$

In the case of $w_{\gamma}^{(-1 / 2)}$, by [61], for $n \geq 4$, we have

$$
\pi_{n}^{(-1 / 2)}(t) \pi_{n+1}^{*(-1 / 2)}(t)=\left(t^{2}-1\right)\left[\pi_{2 n-1}^{(1 / 2)}(t)-\frac{\gamma}{4} \pi_{2 n-3}^{(1 / 2)}(t)\right]
$$

and

$$
\pi_{n}^{(1 / 2)}(t)=\frac{1}{2^{n}}\left[U_{n}(t)-\gamma U_{n-2}(t)\right], \quad U_{n}(\cos \theta)=\sin (n+1) \theta / \sin \theta .
$$

Combining these equalities one obtains

$$
K_{n}^{(-1 / 2)}(z)=-\frac{(1+\gamma)^{2}}{2^{2 n+1} \gamma \pi_{n}^{(-1 / 2)}(z) \pi_{n+1}^{*(-1 / 2)}(z)}\left(A_{2 n-1}-2 \gamma A_{2 n-3}+\gamma^{2} A_{2 n-5}\right),
$$

where

$$
A_{k}=A_{k}(z)=\int_{-1}^{1} \frac{U_{k}(t) \sqrt{1-t^{2}}}{(z-t)\left(t^{2}-\frac{(1+\gamma)^{2}}{4 \gamma}\right)} d t= \begin{cases}\pi \frac{\left(z-\sqrt{z^{2}-1}\right)^{k+1}-\gamma^{\frac{k+1}{2}}}{z^{2}-\frac{(1+\gamma)^{2}}{4 \gamma}}, & k \text { odd }, \\ \pi \frac{\left(z-\sqrt{z^{2}-1}\right)^{k+1}-\frac{2 z}{1+\gamma} \gamma^{\frac{k+2}{2}}}{z^{2}-\frac{(1+\gamma)^{2}}{4 \gamma}}, & k \text { even }\end{cases}
$$

which gives us the explicit expression for $K_{n}^{(-1 / 2)}(z)$.

Similarly, in the case of $w_{\gamma}^{(1 / 2)}$, for $n \geq 2$, we obtain

$$
K_{n}^{(1 / 2)}(z)=-\frac{(1+\gamma)^{2}}{2^{2 n+3} \gamma \pi_{n}^{(1 / 2)}(z) \pi_{n+1}^{*(1 / 2)}(z)}\left(A_{2 n+1}-2 \gamma A_{2 n-1}+\gamma^{2} A_{2 n-3}\right),
$$




\section{ETNA}

Kent State University and

Johann Radon Institute (RICAM)

whereas in the case of $w_{\gamma}^{( \pm 1 / 2, \mp 1 / 2)}$, for $n \geq 3$, we obtain

$$
K_{n}^{( \pm 1 / 2, \mp 1 / 2)}(z)=\mp \frac{(1+\gamma)^{2}\left(A_{2 n}-2 \gamma A_{2 n-2}+\gamma^{2} A_{2 n-4}\right)}{2^{2 n+2} \gamma \pi_{n}^{( \pm 1 / 2, \mp 1 / 2)}(z) \pi_{n+1}^{*( \pm 1 / 2, \mp 1 / 2)}(z)} .
$$

As was the case with the Gauss-Radau formulas, the maximum point of the modulus of the kernel eventually (i.e., whenever $\rho>\rho^{*}=\rho^{*}(n, \gamma)$ ) stabilizes at one of the coordinate axes. The smallest such values of $\rho^{*}$ appear to be close to 1 for all $\gamma \in(-1,0)$ in the case of the weight functions $w_{\gamma}^{(-1 / 2)}$ and $w_{\gamma}^{(\mp 1 / 2, \pm 1 / 2)}$ and smaller than 2 in the case of $w_{\gamma}^{(1 / 2)}$.

In the case of the function $f_{2}(t)=\frac{\cos t}{\alpha^{2}+t^{2}}$ from [61, Example 4.2], we have

$$
\max _{z \in \mathcal{E}_{\rho}}\left|f_{2}(z)\right|=\frac{\cosh b_{1}}{\alpha^{2}-b_{1}^{2}}, \quad b_{1}=\frac{1}{2}\left(\rho-\rho^{-1}\right)
$$

so we must have $b_{1}<\alpha$, i.e., $\rho<\alpha+\sqrt{\alpha^{2}+1}=\rho_{\max }$. The values of the corresponding bounds are compared in Table 4.1.

TABLE 4.1

Comparison of the values $r\left(f_{2}\right)$ in the case of the weight function $w_{\gamma}^{(1 / 2)}$ with the corresponding values from Table 3 in [61].

\begin{tabular}{c|c|c|c|c}
$n, \alpha$ & Bound (4.6) from [61] & Bound (4.7) from [61] & $r\left(f_{2}\right)$ & Error \\
\hline $5,0.75$ & - & - & $1.136(-5)$ & $1.984(-7)$ \\
$10,0.75$ & - & - & $2.129(-11)$ & $1.892(-13)$ \\
$15,0.75$ & - & - & $3.028(-17)$ & $1.805(-19)$ \\
$20,0.75$ & - & - & $3.839(-23)$ & $1.721(-25)$ \\
$5,1.5$ & $1.421(-9)$ & $5.378(-8)$ & $2.425(-10)$ & $3.926(-12)$ \\
$10,1.5$ & $6.210(-18)$ & $4.613(-16)$ & $2.040(-20)$ & $1.646(-22)$ \\
$15,1.5$ & $2.714(-26)$ & $3.006(-24)$ & $1.284(-30)$ & $6.898(-33)$ \\
$20,1.5$ & $1.186(-34)$ & $1.746(-32)$ & $7.184(-41)$ & $2.892(-43)$ \\
$5,3.0$ & $1.166(-16)$ & $3.114(-15)$ & $8.594(-16)$ & $2.846(-17)$ \\
$10,3.0$ & $5.696(-32)$ & $3.160(-30)$ & $2.896(-31)$ & $4.564(-33)$ \\
$15,3.0$ & $2.783(-47)$ & $2.346(-45)$ & $7.083(-47)$ & $7.320(-49)$ \\
$20,3.0$ & $1.360(-62)$ & $1.538(-60)$ & $1.527(-62)$ & $1.174(-64)$ \\
$5,6.0$ & $3.226(-22)$ & $6.822(-21)$ & $4.928(-21)$ & $2.270(-22)$ \\
$10,6.0$ & $9.684(-44)$ & $4.704(-42)$ & $2.591(-42)$ & $5.160(-44)$ \\
$15,6.0$ & $2.907(-65)$ & $2.212(-63)$ & $9.245(-64)$ & $1.173(-65)$ \\
$20,6.0$ & $8.724(-87)$ & $9.042(-85)$ & $2.865(-85)$ & $2.667(-87)$
\end{tabular}

REMARK 4.1. Throughout the last two sections of this paper, we claim that the argument $\theta$ of the extremum point of a kernel eventually (that is, if $\rho$ is large enough, e.g., $\rho \geq \rho^{*}$ ) stabilizes. Although statements of this kind are clearly false in general, in our cases they are justified by a simple principle that is presented below.

We start with a standard lemma.

LEMMA 4.1. Let $P(x, y)=\sum_{i=0}^{n} p_{i}(y) x^{n-i}$ be a continuous function in two variables that is a polynomial in $x$, and assume that $P(x, c) \equiv 0$. If $p_{0}(y)>0$ for $y \in[a, b] \backslash\{c\}$ and, for each $i=1, \ldots, n$, we have $p_{i}(y)=O\left(p_{0}(y)\right)$ for $y$ in a neighborhood of $c$, then there is a constant $M$ such that $P(x, y) \geq 0$ whenever $x \geq M$ and $a \leq y \leq b$. 
Proof. By the hypothesis, there is a constant $M>1$ such that $\left|p_{i}(y)\right| \leq(M-1) p_{0}(y)$ for each $y \in[a, b] \backslash\{c\}$. It follows that

$$
\begin{aligned}
P(x, y) & \geq p_{0}(y) x^{n}-(M-1) \sum_{i=1}^{n} p_{0}(y) x^{n-i} \\
& >p_{0}(y) x^{n}\left(1-\sum_{i=1}^{\infty} \frac{M-1}{x^{i}}\right)=p_{0}(y) x^{n}\left(1-\frac{M-1}{x-1}\right) \geq 0
\end{aligned}
$$

for all $x \geq M$ and $a \leq y \leq b$.

THEOREM 4.1. Let $Q(\rho, \theta)=\sum_{i=0}^{n} q_{i}(\theta) \rho^{n-i}$ and $R(\rho, \theta)=\sum_{i=0}^{m} r_{i}(\theta) \rho^{m-i}$ be continuous functions in two variables that are polynomials in $\rho$. Assume that $R(\rho, \theta)>0$ in the entire region $\rho>K$, and consider the function $f(\rho, \theta)=\frac{Q(\rho, \theta)}{R(\rho, \theta)}$. If

(i) $p_{0}(\theta)=q_{0}\left(\theta_{0}\right) r_{0}(\theta)-q_{0}(\theta) r_{0}\left(\theta_{0}\right)>0$, for $\theta \in[\alpha, \beta] \backslash\left\{\theta_{0}\right\}$, and

(ii) $q_{i}(\theta)-q_{i}\left(\theta_{0}\right)=O\left(p_{0}(\theta)\right)$ and $r_{i}(\theta)-r_{i}\left(\theta_{0}\right)=O\left(p_{0}(\theta)\right)$, for $\theta$ in a neighborhood of $\theta_{0}$ and for each $i=1, \ldots, n$,

then there is a constant $\rho^{*}$ such that for each $\rho \geq \rho^{*}$, we have $\max _{\alpha \leq \theta \leq \beta} f(\rho, \theta)=f\left(\rho, \theta_{0}\right)$.

Proof. Since $f\left(\rho, \theta_{0}\right)-f(\rho, \theta)=\frac{P(\rho, \theta)}{R(\rho, \theta) R\left(\rho, \theta_{0}\right)}$, where

$$
P(\rho, \theta)=Q\left(\rho, \theta_{0}\right) R(\rho, \theta)-Q(\rho, \theta) R\left(\rho, \theta_{0}\right),
$$

it suffices to prove that $P(\rho, \theta)$ is positive for all $\theta \neq \theta_{0}$, whenever $\rho$ is large enough.

The statement will follow from Lemma 4.1 applied to $P(\rho, \theta)$, so it suffices to verify the conditions of the lemma. Indeed, the leading coefficient of $P$ is $p_{0}(\theta)>0$, whereas the coefficient of $\rho^{m+n-k}$ is

$$
\begin{aligned}
p_{k}(\theta) & =\sum_{i+j=k}\left[q_{i}\left(\theta_{0}\right) r_{j}(\theta)-r_{j}\left(\theta_{0}\right) q_{i}(\theta)\right] \\
& =\sum_{i+j=k}\left[q_{i}\left(\theta_{0}\right)\left(r_{j}(\theta)-r_{j}\left(\theta_{0}\right)\right)-r_{j}\left(\theta_{0}\right)\left(q_{i}(\theta)-q_{i}\left(\theta_{0}\right)\right)\right]=O\left(p_{0}(\theta)\right)
\end{aligned}
$$

for $\theta$ in a neighborhood of $\theta_{0}$, and hence the proof is complete.

EXAMPLE 4.2. In [9, Theorem 1], setting $\theta_{0}=0$, we have $p_{0}(\theta)=(1-\cos 2 \theta) \gamma^{5}\left(3 \gamma^{2}+\right.$ $y-1)>0$ if $\frac{-1-\sqrt{13}}{6}<\gamma<0$, so the condition (i) is fulfilled. Moreover, all the coefficients $q_{i}(\theta)-q_{i}(0)$ and $r_{i}(\theta)-r_{i}(0)$ are polynomials in $1-\cos 2 \theta$ and are thus $O(1-\cos 2 \theta)$, as $\theta \rightarrow 0$, so the condition (ii) is fulfilled as well. The other cases and theorems in this paper are verified in the same manner.

5. Error bounds for Gauss-Turán quadratures for analytic functions-a general method. Let $d \mu(t)=w(t) d t$ be a nonnegative measure (distribution) supported on the interval $(-1,1)$, where $w$ is an integrable weight function on this interval. We consider the Gauss-Turán quadrature formulas with multiple nodes:

$$
\int_{-1}^{1} f(t) d \mu(t)=Q_{n, s}[f]+R_{n, s}(f), \quad Q_{n, s}[f]=\sum_{\nu=1}^{n} \sum_{i=0}^{2 s} A_{i, \nu} f^{(i)}\left(\tau_{\nu}\right) .
$$

Quadrature formulas with multiple nodes appeared more than 100 years after the renowned Gaussian quadratures. A well-known subclass thereof are the Gauss-Turán quadrature formulas with all nodes having the same multiplicity; see [94].

In [89], following an approach of von Sydow [95] and de la Calle Ysern [7] (see also [8]) for Gaussian and rational quadrature formulas, respectively, we presented a computable upper 
bound for the error which is valid for a broad range of Gauss-Turán quadrature formulas with arbitrary weight functions.

Since $\mathcal{E}_{\rho}(\rho>1)$ given by (1.6) shrinks to the interval $[-1,1]$ as $\rho$ tends to $1+$, there is a maximal parameter $\rho_{\max }$ such that $f$ is analytic inside $\mathcal{E}_{\rho}$ whenever $1<\rho<\rho_{\max }$. The contour integral representation then reads

$$
R_{n, s}(f)=\frac{1}{2 \pi i} \oint_{\mathcal{E}_{\rho}} K_{n ; s}(z) f(z) d z, \quad 1<\rho<\rho_{\max },
$$

where the kernel $K_{n ; s}$ is given by

$$
\begin{aligned}
K_{n, s}(z) & =R_{n, s}\left(\frac{1}{z-\cdot}\right)=\int_{-1}^{1} \frac{1}{z-t} d \mu(t)-\sum_{\nu=1}^{n} \sum_{i=0}^{2 s} \frac{i ! A_{i, \nu}}{\left(z-\tau_{\nu}\right)^{i+1}} \\
& =\int_{-1}^{1} \frac{1}{z-t} d \mu(t)-\sum_{\nu=1}^{n} \frac{A_{0, \nu}}{z-\tau_{\nu}}-\sum_{\nu=1}^{n} \sum_{i=1}^{2 s} \frac{i ! A_{i, \nu}}{\left(z-\tau_{\nu}\right)^{i+1}} .
\end{aligned}
$$

From (5.1), for an arbitrary polynomial $S_{2 n(s+1)-1} \in \mathcal{P}_{2 n(s+1)-1}$, we get

$$
\begin{aligned}
R_{n, s}(f) & =\int_{-1}^{1} f(t) d \mu(t)-Q_{n, s}[f] \\
& =\int_{-1}^{1}\left[f(t)-S_{2 n(s+1)-1}(t)\right] d \mu(t)+Q_{n, s}\left[S_{2 n(s+1)-1}-f\right] .
\end{aligned}
$$
bounds

Taking $f(t)=1 /(z-t)\left(z \in \mathcal{E}_{\rho}, t \in[-1,1]\right)$, the above formula gives us the upper

$$
\begin{aligned}
\left|R_{n, s}\left(\frac{1}{z-t}\right)\right| \leq \quad \int_{-1}^{1} & \left|\frac{1}{z-t}-S_{2 n(s+1)-1}(t)\right| d \mu(t) \\
& +\sum_{\nu=1}^{n} A_{0, \nu}\left|\frac{1}{z-\tau_{\nu}}-S_{2 n(s+1)-1}\left(\tau_{\nu}\right)\right| \\
& +\left|\sum_{\nu=1}^{n} \sum_{i=1}^{2 s} A_{i, \nu} \cdot\left(\frac{i !}{\left(z-\tau_{\nu}\right)^{i+1}}-S_{2 n(s+1)-1}^{(i)}\left(\tau_{\nu}\right)\right)\right|
\end{aligned}
$$

and

$$
\begin{aligned}
\left|R_{n, s}\left(\frac{1}{z-t}\right)\right| \leq \int_{-1}^{1} & \left|\frac{1}{z-t}-S_{2 n(s+1)-1}(t)\right| d \mu(t) \\
& +\sum_{\nu=1}^{n} A_{0, \nu}\left|\frac{1}{z-\tau_{\nu}}-S_{2 n(s+1)-1}\left(\tau_{\nu}\right)\right| \\
& +\sum_{\nu=1}^{n} \sum_{i=1}^{2 s}\left|A_{i, \nu}\right| \cdot\left|\frac{i !}{\left(z-\tau_{\nu}\right)^{i+1}}-S_{2 n(s+1)-1}^{(i)}\left(\tau_{\nu}\right)\right| .
\end{aligned}
$$

Here we used the fact that the weights $A_{0, \nu}$ are non-negative; see e.g., [71, p. 103].

Let us denote by $\tilde{S}_{2 n(s+1)-1}$ the best min-max polynomial approximation of the function $1 /(z-t)$ on the interval $[-1,1]$, i.e.,

$$
\begin{aligned}
\max _{t \in[-1,1]}\left|\frac{1}{z-t}-\tilde{S}_{2 n(s+1)-1}(t)\right| & =\min _{S \in \mathcal{P}_{2 n(s+1)-1}} \max _{t \in[-1,1]}\left|\frac{1}{z-t}-S(t)\right| \\
& =\widetilde{\mathcal{E}}_{2 n(s+1)-1}\left(\frac{1}{z-t}\right) .
\end{aligned}
$$


From (5.4) we get

$$
\begin{aligned}
\left|R_{n, s}\left(\frac{1}{z-t}\right)\right| \leq & 2 \mu_{0} \cdot \widetilde{\mathcal{E}}_{2 n(s+1)-1}\left(\frac{1}{z-t}\right) \\
& +\sum_{\nu=1}^{n} \sum_{i=1}^{2 s}\left|A_{i, \nu}\right| \cdot\left|\frac{i !}{\left(z-\tau_{\nu}\right)^{i+1}}-\tilde{S}_{2 n(s+1)-1}^{(i)}\left(\tau_{\nu}\right)\right|,
\end{aligned}
$$

where $\mu_{0}=\int_{-1}^{1} d \mu(t)$. Let us define

$$
\Phi_{0,2 n(s+1)-1}(t)=\frac{1}{z-t}-\tilde{S}_{2 n(s+1)-1}(t) .
$$

It is well known (cf. [95], [1]) that

$$
\Phi_{0,2 n(s+1)-1}(t) \equiv G(v)=\frac{M}{2}\left(v^{2 n(s+1)-1} \cdot \frac{\bar{\alpha}-v}{1-\alpha v}+v^{-2 n(s+1)+1} \cdot \frac{1-\bar{\alpha} v}{\alpha-v}\right),
$$

with

$$
|M|=\widetilde{\mathcal{E}}_{2 n(s+1)-1}\left(\frac{1}{z-t}\right) \leq \frac{2\left|z-\sqrt{z^{2}-1}\right|^{2 n(s+1)}}{\left|\sqrt{z^{2}-1}\right| \cdot\left(1-\left|z-\sqrt{z^{2}-1}\right|^{2}\right)}
$$

and

$$
t=\frac{1}{2}\left(v+\frac{1}{v}\right), \quad z=\frac{1}{2}\left(\alpha+\frac{1}{\alpha}\right), \quad \alpha=z-\sqrt{z^{2}-1}, \quad|\alpha|=\rho^{-1} .
$$

Therefore,

$$
|M| \leq \frac{2 \rho^{-2 n(s+1)}}{\left|\sqrt{z^{2}-1}\right| \cdot\left(1-\rho^{-2}\right)} .
$$

Recall that $t=\cos \theta \in[-1,1], v=v(t)=e^{i \theta}, \theta \in[0, \pi]$, with $\tau_{\nu}=-\cos \theta_{\nu}$ $(\nu=1, \ldots, n)$. The upper bound (5.5) can now be written as

$$
\left|R_{n, s}\left(\frac{1}{z-t}\right)\right| \leq 2 \mu_{0} \cdot \widetilde{\mathcal{E}}_{2 n(s+1)-1}\left(\frac{1}{z-t}\right)+\sum_{\nu=1}^{n} \sum_{i=1}^{2 s}\left|A_{i, \nu}\right| \cdot\left|\Phi_{0,2 n(s+1)-1}^{(i)}\left(\tau_{\nu}\right)\right| .
$$

Alternatively, if we want to reduce the number of numerical calculations, we can use a cruder bound:

$$
\left|R_{n, s}\left(\frac{1}{z-t}\right)\right| \leq 2 \mu_{0} \cdot \widetilde{\mathcal{E}}_{2 n(s+1)-1}\left(\frac{1}{z-t}\right)+\sum_{i=1}^{2 s} \sum_{\nu=1}^{n} D_{i, \nu} \cdot\left|A_{i, \nu}\right|,
$$

where $\left|\Phi_{0,2 n(s+1)-1}^{(i)}\left(\tau_{\nu}\right)\right| \leq D_{i, \nu}$, for $\nu=1, \ldots, n$ and $i=1, \ldots, 2 s$.

In [89] we focused on the error bounds based on the inequality of the type (5.8), the effectiveness thereof was shown there. The parameters $\tau_{\nu}, A_{i, \nu}$ are calculated numerically by the stable numerical method given by Gautschi and Milovanović (see [18]; also [48, 85]). In fact, for these calculations we used the Matlab templates turan.m, sturan.m which are downloadable from the Web site [14] and distributed along with the book [13]. 
Determining the quantities $\Phi_{0,2 n(s+1)-1}^{(i)}(t)$ and $D_{i, \nu}$ is possible, although it gets complicated for larger $i$. For a given $s \in \mathbb{N}$, we have to find the derivatives $\Phi_{0,2 n(s+1)-1}^{(i)}(t)$ for $i=0,1, \ldots, 2 s$, which are of the form

$$
\begin{aligned}
\Phi_{0,2 n(s+1)-1}^{(i)}(t) & =\frac{d^{i}}{d t^{i}} \Phi_{0,2 n(s+1)-1}(t)=\frac{d^{i}}{d t^{i}}(G(v(t)) \\
& =\sum_{k=1}^{i} G^{(k)}(v) \cdot \sum_{k_{1}, k_{2}, \ldots, k_{i}} C_{k_{1}, k_{2}, \ldots, k_{i}} \cdot \prod_{l=1}^{i}\left[v_{t}^{(l)}(t)\right]^{k_{l}},
\end{aligned}
$$

where the latter sum is taken over certain combinations of $k_{1}, k_{2}, \ldots, k_{i}(\in\{0,1, \ldots, i\})$, $C_{k_{1}, k_{2}, \ldots, k_{i}}$ are the corresponding real constants, and $v_{t}^{(l)}(t)=d^{l} v(t) / d t^{l}$. Now we can obtain the upper bounds

$$
\left|\Phi_{0,2 n(s+1)-1}^{(i)}\left(\tau_{\nu}\right)\right| \leq \sum_{k=1}^{i}\left|G^{(k)}\left(v\left(\tau_{\nu}\right)\right)\right| \cdot \sum_{k_{1}, k_{2}, \ldots, k_{i}}\left|C_{k_{1}, k_{2}, \ldots, k_{i}}\right| \cdot \prod_{l=1}^{i}\left|v_{t}^{(l)}\left(\tau_{\nu}\right)\right|^{k_{l}} .
$$

By Leibnitz's formula, (5.6) gives us

$$
G^{(k)}(v)=\frac{M}{2} \sum_{j=0}^{k}\left(\begin{array}{l}
k \\
j
\end{array}\right)\left[f_{1}^{(j)}(v) g_{1}^{(k-j)}(v)+f_{2}^{(j)}(v) g_{2}^{(k-j)}(v)\right]
$$

where

$$
f_{1}(v)=\frac{\bar{\alpha}-v}{1-\alpha v}, \quad g_{1}(t)=v^{2 n(s+1)-1}, \quad f_{2}(v)=\frac{1-\bar{\alpha} v}{\alpha-v}, \quad g_{2}(v)=v^{-2 n(s+1)+1}
$$

and

$$
\left|G^{(k)}(v)\right| \leq \frac{|M|}{2} \sum_{j=0}^{k}\left(\begin{array}{l}
k \\
j
\end{array}\right)\left[\left|f_{1}^{(j)}(v)\right| \cdot\left|g_{1}^{(k-j)}(v)\right|+\left|f_{2}^{(j)}(v)\right| \cdot\left|g_{2}^{(k-j)}(v)\right|\right] .
$$

For $j \in \mathbb{N}$, we have

$$
\begin{aligned}
& f_{1}^{(j)}(v)=j ! \alpha^{j-1}\left(-1+|\alpha|^{2}\right)(1-\alpha v)^{-j-1} \\
& f_{2}^{(j)}(v)=j ! \alpha^{j-1}\left(1-|\alpha|^{2}\right)(\alpha-v)^{-j-1}
\end{aligned}
$$

and for $j<k$, we have

$$
\begin{aligned}
& g_{1}^{(k-j)}(v)=(2 n(s+1)-1)(2 n(s+1)-2) \cdots(2 n(s+1)-k+j) \cdot v^{2 n(s+1)-k+j-1}, \\
& g_{2}^{(k-j)}(v)=(-1)^{k-j}(2 n(s+1)-1)(2 n(s+1)) \cdots(2 n(s+1)+k-j-2) \\
& \quad \times v^{-2 n(s+1)-k+j+1} .
\end{aligned}
$$

Furthermore, for $j \in \mathbb{N}$, we deduce

$$
\begin{aligned}
& \left|f_{1}^{(j)}(v)\right| \leq j !|\alpha|^{j-1}\left(1-|\alpha|^{2}\right)(1-|\alpha|)^{-j-1}, \\
& \left|f_{2}^{(j)}(v)\right| \leq j !|\alpha|^{j-1}\left(1-|\alpha|^{2}\right)(1-|\alpha|)^{-j-1},
\end{aligned}
$$


and, for $j<k$,

$$
\begin{aligned}
& \left|g_{1}^{(k-j)}(v)\right|=(2 n(s+1)-1)(2 n(s+1)-2) \cdots(2 n(s+1)-k+j), \\
& \left|g_{2}^{(k-j)}(v)\right|=(2 n(s+1)-1)(2 n(s+1)) \cdots(2 n(s+1)+k-j-2) .
\end{aligned}
$$

Now the inequalities (5.10) obtain the form

$$
\left|G^{(k)}(v)\right| \leq \frac{|M|}{2} \cdot L_{k} \quad(k \geq 1),
$$

where

$$
\begin{aligned}
L_{k}= & k !\left\{\left(\begin{array}{c}
2 n(s+1)-1 \\
k
\end{array}\right)+\left(\begin{array}{c}
2 n(s+1)+k-2 \\
k
\end{array}\right)\right. \\
& \left.+(\rho+1) \sum_{j=1}^{k}\left[\left(\begin{array}{c}
2 n(s+1)-1 \\
k-j
\end{array}\right)+\left(\begin{array}{c}
2 n(s+1)+k-j-2 \\
k-j
\end{array}\right) \rho^{j-1}\right] \cdot \frac{1}{(\rho-1)^{j}}\right\} .
\end{aligned}
$$

The quantities $\left|v_{t}^{(l)}\left(\tau_{\nu}\right)\right|=: V_{l, \nu}$ in (5.9) can be evaluated as follows. As $v(t)=t-$ $\sqrt{t^{2}-1}$, we have that $v_{t}^{\prime \prime}=\left(t^{2}-1\right)^{-3 / 2}=(t-1)^{-3 / 2}(t+1)^{-3 / 2}$. For $l \geq 3$, applying Leibnitz's formula yields

$$
\begin{aligned}
v_{t}^{(l)} & =\left(v_{t}^{\prime \prime}\right)^{(l-2)}=\left((t-1)^{-3 / 2}(t+1)^{-3 / 2}\right)^{(l-2)} \\
& =\sum_{j=0}^{l-2}\left(\begin{array}{c}
l-2 \\
j
\end{array}\right)\left((t+1)^{-3 / 2}\right)^{(l-2-j)}\left((t-1)^{-3 / 2}\right)^{(j)} \\
& =\frac{(-1)^{l}}{2^{l-2}}\left(t^{2}-1\right)^{-\frac{2 l-1}{2}} \sum_{j=0}^{l-2}\left(\begin{array}{c}
l-2 \\
j
\end{array}\right)(2(l-2-j)+1) ! !(2 j+1) ! !(t+1)^{j}(t-1)^{l-2-j},
\end{aligned}
$$

where $j ! !=j ! /(j-1) !$. Therefore,

$$
\begin{aligned}
V_{l, \nu}= & \frac{1}{2^{l-2}}\left(1-\tau_{\nu}^{2}\right)^{-\frac{2 l-1}{2}} \\
& \times\left|\sum_{j=0}^{l-2}\left(\begin{array}{c}
l-2 \\
j
\end{array}\right)(2(l-2-j)+1) ! !(2 j+1) ! !\left(\tau_{\nu}+1\right)^{j}\left(\tau_{\nu}-1\right)^{l-2-j}\right|,
\end{aligned}
$$

where $\nu=1,2, \ldots, n$ and $l=3,4, \ldots, 2 s$. Finally, knowing that

$$
\int_{\mathcal{E}_{\rho}} \frac{|d z|}{\left|\sqrt{z^{2}-1}\right|}=2 \pi
$$

on the basis of (5.7), by using (5.9), (5.10), (5.11), (5.12), the error bound of the type (5.8) in the general case $(s \in \mathbb{N})$ obtains the form

$$
\left|R_{n, s}(f)\right| \leq e_{n, s}(f)
$$

where

$$
\begin{aligned}
e_{n, s}(f)= & \inf _{1<\rho<\rho_{\max }}\left\{\left(\max _{z \in \mathcal{E}_{\rho}}|f(z)|\right) \cdot \rho^{-2 n(s+1)}\left(1-\rho^{-2}\right)^{-1}\right. \\
& \left.\times\left[4 \mu_{0}+\sum_{i=1}^{2 s} \sum_{k=1}^{i} L_{k} \sum_{k_{1}, k_{2}, \ldots, k_{i}}\left|C_{k_{1}, k_{2}, \ldots, k_{i}}\right| \cdot \sum_{\nu=1}^{n} \prod_{l=1}^{i}\left|A_{i, \nu}\right| V_{l, \nu}^{k_{l}}\right]\right\},
\end{aligned}
$$


and $L_{k}, V_{i, \nu}$ are given by (5.11), (5.12), respectively.

There are several papers about bounding $\left|A_{i, \nu}\right|$; cf. [71, 96]. See [89] for more details. In [89] we demonstrated how to determine $e_{n, s}(f)$ in (5.13) in the cases $s=1,2$. Here is what we obtained for $s=2$ :

$$
\left|R_{n, 2}(f)\right| \leq e_{n, 2}(f)
$$

where

$$
\begin{aligned}
e_{n, 2}(f)= & \inf _{1<\rho<\rho_{\max }}\left\{\left(\max _{z \in \mathcal{E}_{\rho}}|f(z)|\right) \cdot 2 \rho^{-6 n}\left(1-\rho^{-2}\right)^{-1}\right. \\
& \times\left[2 \mu_{0}+\left(2 n(s+1)-1+\frac{\rho+1}{\rho-1}\right) \sum_{\nu=1}^{n}\left(\frac{\left|A_{1, \nu}\right|}{\left(1-\tau_{\nu}^{2}\right)^{1 / 2}}+\frac{\left|A_{2, \nu}\right|}{\left(1-\tau_{\nu}^{2}\right)^{3 / 2}}\right)\right. \\
& +\left(2 n(s+1)-1+\frac{\rho+1}{\rho-1}\right)^{2} \sum_{\nu=1}^{n} \frac{\left|A_{2, \nu}\right|}{1-\tau_{\nu}^{2}} \\
& +\left(\left(2 n(s+1)-1+\frac{\rho+1}{\rho-1}\right)^{3}+2(2 n(s+1)-1)+2 \frac{\rho^{3}+1}{(\rho-1)^{3}}\right) \sum_{\nu=1}^{n} \frac{\left|A_{3, \nu}\right|}{\left(1-\tau_{\nu}^{2}\right)^{3 / 2}} \\
& +3\left(2 n(s+1)-1+\frac{\rho+1}{\rho-1}\right)^{2} \sum_{\nu=1}^{n}\left(\frac{\left|A_{3, \nu}\right|}{\left(1-\tau_{\nu}^{2}\right)^{2}}+\frac{\left|A_{4, \nu}\right|}{\left(1-\tau_{\nu}^{2}\right)^{3}} \cdot\left(1+4\left|\tau_{\nu}\right|\right)\right) \\
& +3\left(2 n(s+1)-1+\frac{\rho+1}{\rho-1}\right)^{n} \sum_{\nu=1}^{n}\left(\left|A_{3, \nu}\right| \frac{\left|\tau_{\nu}\right|}{\left(1-\tau_{\nu}^{2}\right)^{5 / 2}}+\left|A_{4, \nu}\right| \frac{1+4 \tau_{\nu}^{2}}{\left(1-\tau_{\nu}^{2}\right)^{7 / 2}}\right) \\
& +\left(\left(2 n(s+1)-1+\frac{\rho+1}{\rho-1}\right)^{4}\right. \\
& +(2 n(s+1)-1)\left(11(2 n(s+1)-1)+14 \frac{\rho+1}{\rho-1}+8 \frac{\rho^{3}+1}{(\rho-1)^{3}}\right) \\
& \left.+12 \frac{(\rho+1)\left(\rho^{3}+1\right)}{(\rho-1)^{4}}-\left(\frac{\rho+1}{\rho-1}\right)^{4}\right) \sum_{\nu=1}^{n} \frac{\left|A_{4, \nu}\right|}{\left(1-\tau_{\nu}^{2}\right)^{2}} \\
& +6\left(\left(2 n(s+1)-1+\frac{\rho+1}{\rho-1}\right)^{3}+2(2 n(s+1)-1)+2 \frac{\rho^{3}+1}{(\rho-1)^{3}}\right) \\
& \left.\left.\sum_{\nu=1}^{n} \frac{\left|A_{4, \nu}\right|}{\left(1-\tau_{\nu}^{2}\right)^{5 / 2}}\right]\right\} \cdot
\end{aligned}
$$

The general error bound $e_{n, s}(f)$ in (5.13) has a rather complicated form. The reason for this is the impossibility of determining the derivative $d_{k}(t)=\left(G(v(t))_{t}^{(k)}\right.$ in some condensed form for arbitrary $k \in \mathbb{N}$. On the other hand, as is well known, determining $d_{i}(t)$ formally is quite simple, although it requires patience. We have $d_{0}(t)=G(v(t)), d_{1}(t)=G^{\prime}(v) \cdot v_{t}^{\prime}$, and $d_{k}(t)=\left(d_{k-1}(t)\right)_{t}^{\prime}$, for $k=2,3, \ldots, 2 s$. This is the first step in the algorithm that has been used to handle (5.13) by the computer. Next, we use the given form (5.6) of $G(v)$. The next step is bounding $\left|G^{(k)}(v)\right|$ by $|M| L_{k} / 2$, independently of $v,|v|=1$ (and therefore of $t$, $t \in[-1,1])$, where the $L_{k}$ are given by (5.11). The calculations to this point do not depend on the Gauss-Turán quadrature under consideration, i.e., its nodes and weights. Finally, we calculate the nodes $\tau_{\nu}$ and the weights $A_{i, \nu}$ of the given Gauss-Turán quadrature and the 
quantities $V_{l, \nu}=\left|v_{t}^{(l)}\left(\tau_{\nu}\right)\right|$ by (5.12) and form the function whose infimum is required on $\left(1, \rho_{\max }\right)$; see the formula for $e_{n, s}(f)$ in (5.13).

From [96, Theorem 3.1] and (5.3) for large values of $n$, we deduce that

$$
\left|R_{n, s}\left(\frac{1}{z-t}\right)\right| \sim 2 \mu_{0} \cdot \widetilde{\mathcal{E}}_{2 n(s+1)-1}\left(\frac{1}{z-t}\right) \quad(n \rightarrow \infty) .
$$

By using the above equation for large values of $n$, we get from (5.2) the asymptotic error estimate

$$
\left|R_{n, s}(f)\right| \sim e_{n, s}^{(\mathbf{a})}(f ; w)(n \rightarrow \infty)
$$

where

$$
e_{n, s}^{(\mathbf{a})}(f ; w)=\inf _{1<\rho<\rho_{\max }}\left\{\left(4 \mu_{0}\left(1-\rho^{-2}\right)^{-1} \rho^{-2 n(s+1)}\right) \cdot\left(\max _{z \in \mathcal{E}_{\rho}}|f(z)|\right)\right\} .
$$

The asymptotic error estimate $\left|R_{n, s}(f)\right| \sim e_{n, s}^{(\text {as })}(f)(n \rightarrow \infty)$, where

$$
\begin{aligned}
e_{n, s}^{(\mathbf{a s})}(f) & =\inf _{1<\rho<\rho_{\max }}\left\{\rho^{-2 n(s+1)}\left(1-\rho^{-2}\right)^{-1} \times\right. \\
& \left.\times\left(4 \mu_{0}+\frac{1}{\pi} \sum_{\nu=1}^{n} \sum_{i=1}^{2 s} i !\left|A_{i, \nu}\right|\left(1-\rho^{-2}\right)^{-i} \cdot \oint_{\mathcal{E}_{\rho}} \frac{|d z|}{\left|\sqrt{z^{2}-1}\right|^{i+1}}\right)\left(\max _{z \in \mathcal{E}_{\rho}}|f(z)|\right)\right\},
\end{aligned}
$$

has been also proposed in [89]. The above integral can be computed explicitly (cf. Gradshteyn and Ryzhik [25]), but the computation gets complicated for larger values of $i$.

A couple of numerical examples showing the efficiency of our methods are given in [89]. Here we only present one.

EXAMPLE 5.1. Denote by "Error" the sharp (actual) error bound of the corresponding Gauss-Turán quadrature formula. Denote by $e_{n, s}(f) \equiv e_{n, s}(f ; w)$ our error bound in order to stress the dependence on the weight function. Let us consider the Gauss-Turán quadrature formula with the Legendre measure $d \mu(t)=w_{0,0}(t), d t=d t$, for the integrand $f(t)=e^{-\omega t^{2}}$, $\omega>0$. The function $f(z)$ is entire and it is easy to see that

$$
\max _{z \in \mathcal{E}_{\rho}}\left|e^{-\omega z^{2}}\right|=e^{\omega b_{1}^{2}}, \quad b_{1}=\frac{1}{2}\left(\rho-\rho^{-1}\right) .
$$

Some results for $s=2$ are displayed in Table 5.1.

Besides the generalized Chebyshev weight functions (1.7), the Gori-Micchelli ones are also important in the theory of Gauss-Turán quadratures. Gori and Micchelli [24] introduced for each $n$ a class of weight functions defined on $[-1,1]$ for which explicit Gauss-Turán quadrature formulas can be found for all $s$. These classes of weight functions have the peculiarity that the corresponding orthogonal polynomials, well-known as $s$-orthogonal polynomials, of the same degree, are independent of $s$. This class includes certain generalized Jacobi weight functions

$$
w_{n, \mu}(t)=\left|U_{n-1}(t) / n\right|^{2 \mu+1}\left(1-t^{2}\right)^{\mu},
$$

where $U_{n-1}(\cos \theta)=\sin n \theta / \sin \theta$ (Chebyshev polynomial of the second kind) and $\mu>-1$. In this case, the Chebyshev polynomials of the first kind $T_{n}(t)$ are $s$-orthogonal polynomials. 
TABLE 5.1

Error bounds $e_{n, 2}\left(f ; w_{0,0}\right)$ and actual errors.

\begin{tabular}{c|c|c|c|r|c|c|c}
$\omega$ & $n$ & $e_{n, 2}\left(f ; w_{0,0}\right)$ & Error & $\omega$ & $n$ & $e_{n, 2}\left(f ; w_{0,0}\right)$ & Error \\
\hline 0.05 & 3 & $2.966(-20)$ & $5.143(-22)$ & 0.01 & 3 & $1.537(-26)$ & $2.683(-28)$ \\
& 4 & $4.848(-29)$ & $7.985(-31)$ & & 4 & $2.015(-37)$ & $3.333(-39)$ \\
& 5 & $3.648(-38)$ & $5.844(-40)$ & & 5 & $1.215(-48)$ & $1.966(-50)$ \\
& 6 & $1.546(-47)$ & $2.395(-49)$ & & 6 & $4.121(-60)$ & $6.400(-62)$ \\
0.5 & 3 & $2.475(-11)$ & $4.180(-13)$ & 0.1 & 3 & $1.491(-17)$ & $2.573(-19)$ \\
& 4 & $3.988(-17)$ & $6.457(-19)$ & & 4 & $1.946(-25)$ & $3.194(-27)$ \\
& 5 & $2.978(-23)$ & $4.745(-25)$ & & 5 & $1.170(-33)$ & $1.883(-35)$ \\
& 6 & $1.256(-29)$ & $1.928(-31)$ & & 6 & $3.962(-42)$ & $6.128(-44)$ \\
& 3 & $3.642(-6)$ & $5.582(-8)$ & 1 & 3 & $1.027(-8)$ & $1.705(-10)$ \\
& 4 & $3.441(-10)$ & $5.407(-12)$ & & 4 & $1.308(-13)$ & $2.093(-15)$ \\
& 5 & $1.611(-14)$ & $2.513(-16)$ & & 5 & $7.759(-19)$ & $1.227(-20)$ \\
& 6 & $4.293(-19)$ & $6.485(-21)$ & & 6 & $2.607(-24)$ & $3.976(-26)$
\end{tabular}

In the special case when the weight function is one of the four generalized Chebyshev weights (1.7), for Gauss-Turán quadrature formulae (5.1) (which are the main representative of quadratures with multiple nodes), we investigated in detail error bounds of the type (1.3) (with $\Gamma=\mathcal{E}_{\rho}$ )—following the approach form [20, 21, 84]—in [39, 49], and error bounds of the type (1.4) (with $\Gamma=\mathcal{E}_{\rho}$ ), jointly with error bounds based on an expansion of the remainder term into a series - following the approaches form [28]—in [42]. So, in [42], on the basis of the proved cases $s=1$ and $s=2$ for the Gauss-Turán quadrature with the Chebyshev weight function of the first kind $w_{1}(t)=1 / \sqrt{1-t^{2}}$, we conjectured the following statement for arbitrary $s \in \mathbb{N}$, which was later verified in [37].

THEOREM 5.1. For the Gauss-Turán quadrature formula (5.1) with the Chebyshev weight function of the first kind $w_{1}(t)=1 / \sqrt{1-t^{2}}$, if the function $f$ is analytic in the interior $\mathcal{D}$ of the ellipse $\mathcal{E}_{\rho}$ and continuous in $\overline{\mathcal{D}}$, then there holds the following error bound

$$
\left|R_{n, s}(f)\right| \leq 2 \pi\left(\max _{z \in \mathcal{E}_{\rho}}|f(z)|\right) \frac{\sum_{k=0}^{s}(-1)^{k}\left(\begin{array}{c}
2 s+1 \\
s-k
\end{array}\right) \rho^{2 n(s-k)}}{\left(\rho^{2 n}-1\right)^{2 s+1}} .
$$

In the meantime, the methods from [39, 49], [42] have been considered not only for Gauss-Turán quadrature formulae (including ones for evaluating integrals with strong singularities [44]), but also for their Radau, Lobatto, and Kronrod extensions, with generalized Chebyshev and Gori-Micchelli weight functions; see [40, 43, 44, 45, 52, 53, 34].

In [47] we analyzed quadrature formulas of high degree of precision for computing Fourier coefficients in expansions of functions with respect to a system of orthogonal polynomials, started by Bojanov and Petrova [6], and we extended their results. The construction of new Gaussian quadrature formulas for the Fourier coefficients of a function, based on the values of the function and its derivatives, is considered there. We proved the existence and uniqueness of Kronrod extensions with multiple nodes of standard Gaussian quadrature formulas with multiple nodes for several weight functions in order to construct some new generalizations of quadrature formulas for the Fourier coefficients.

For the quadrature formulas for the Fourier coefficients based on the zeros of the corresponding orthogonal polynomials, we constructed Kronrod extensions with multiple nodes 
and the highest algebraic degree of precision. Gaussian quadrature formulas, relative to the Chebyshev weight functions, with multiple nodes and their optimal extensions for computing the Fourier coefficients in expansions of functions with respect to a system of orthogonal polynomials, are considered in [36]. The existence and uniqueness of such quadratures is proved. One of them is a generalization of the well-known Micchelli-Rivlin quadrature formula. The others are new. A numerically stable construction of these quadratures is proposed in [36]. The methods from [39, 49], [42] have been considered for such quadrature-type formulas; see [54, 78, 79, 81]. In Section 6 we describe briefly the methods from [54].

6. Error bounds for Kronrod's extensions of a generalization of the Micchelli-Rivlin quadrature formula for analytic functions. The Gaussian quadrature formula with multiple nodes

$$
\int_{-1}^{1} f(t) T_{n}(t) \frac{d t}{\sqrt{1-t^{2}}}=\sum_{\nu=1}^{n} \sum_{i=0}^{2 s-1} A_{i, \nu} f^{(i)}\left(\tau_{\nu}\right)+R_{n, s}\left(f T_{n}\right)
$$

for calculating the Fourier-Chebyshev coefficients of an analytic function $f$, where $n, s \in \mathbb{N}$, with respect to the Chebyshev weight function of the first kind $w(t)=1 / \sqrt{1-t^{2}}$ was introduced in [6, p. 383], and then examined in more detail in [36]. Here $T_{n}$ is the Chebyshev polynomial of the first kind of degree $n$, and the nodes $\tau_{\nu}$ are its zeros. The quadrature rule has the algebraic degree of precision $n(2 s+1)-1$. The special case $s=1$ of (6.1) represents the well-known Micchelli-Rivlin quadrature formula; see [31].

In [36] we considered the Kronrod extension of (6.1) in the form

$$
\int_{-1}^{1} f(t) T_{n}(t) \frac{d t}{\sqrt{1-t^{2}}}=\sum_{\nu=1}^{n} \sum_{i=0}^{2 s-1} B_{i, \nu} f^{(i)}\left(\tau_{\nu}\right)+\sum_{j=1}^{n+1} C_{j} f\left(\hat{\tau}_{j}\right)+R_{n, s}\left(f T_{n}\right),
$$

which has the algebraic degree of precision $2 s n+2 n+1$. The nodes $\tau_{\nu}$ are the same as in (6.1), and the $\hat{\tau}_{j}$ are the zeros of the monic polynomial

$$
F_{n+1}(t)=\frac{1}{2^{n}}\left(T_{n+1}(t)-T_{n-1}(t)\right)=\frac{1}{2^{n-1}}\left(t^{2}-1\right) U_{n-1}(t),
$$

where $U_{n-1}$ is the Chebyshev polynomial of the second kind of degree $n-1$.

Error bounds for the Micchelli-Rivlin quadrature formulas, and then for (6.1), for functions analytic on confocal ellipses that contain the interval $[-1,1]$ in the interior, have been considered in $[78,81]$, respectively. In this paper, our aim is to do the same for the quadrature formulas (6.2).

Let $f$ be an analytic function in a domain $D$ which contains the interval $[-1,1]$ in its interior, and let $\Gamma$ be a simple closed curve in $D$ surrounding $[-1,1]$. Assume that we know the values of the function $f$ and its derivatives $f^{(i)}, i=1,2, \ldots, 2 s-1$, at the zeros of the polynomial $T_{n}$, and that we also know the values of the function $f$ at the zeros of the polynomial $\left(1-t^{2}\right) U_{n-1}(t)$.

The contour integral representation of the remainder term in (6.2) is (see [54])

$$
R_{n, s}\left(f T_{n}\right)=\frac{1}{2 \pi i} \oint_{\Gamma} K_{n, s}(z) f(z) d z,
$$

where the kernel is given by

$$
K_{n, s}(z)=\frac{\varrho_{n, s}(z)}{\left(1-z^{2}\right) T_{n}^{2 s}(z) U_{n-1}(z)}
$$


and

$$
\varrho_{n, s}(z)=\int_{-1}^{1} \frac{w(t)}{z-t}\left(1-t^{2}\right) T_{n}^{2 s+1}(t) U_{n-1}(t) d t .
$$

We take the contour $\Gamma$ to be an ellipse $\mathcal{E}_{\rho}$ as in the previous section.

In the sequel we present three types of bounds, $L^{\infty}$-error bounds (1.3), $L^{1}$-error bounds (1.4), and the error bounds resulting from expanding the remainder term. Numerical examples that illustrate these three estimates are also included. In order to express the kernel (6.3) explicitly, we evaluate the integral (6.4). After some straightforward calculations, we get

$$
K_{n, s}(z)=2 \pi \frac{\sum_{k=0}^{s}\left(\left(\begin{array}{c}
2 s+1 \\
s-k-1
\end{array}\right)-\left(\begin{array}{c}
2 s+1 \\
s-k
\end{array}\right)\right) u^{-2 k n-2 n}}{\left(u-u^{-1}\right)\left(u^{n}+u^{-n}\right)^{2 s}\left(u^{n}-u^{-n}\right)} .
$$

We use the same method as in the previous section and Remark 4.1 and get the following result; see [54].

THEOREM 6.1. For each $n \in \mathbb{N}, n>1$, and each $s \in \mathbb{N}$, there exists $\rho_{0}=\rho_{0}(n, s)$ such that

$$
\max _{z \in \mathcal{E}_{\rho}}\left|K_{n, s}(z)\right|=\left|K_{n, s}\left(\frac{1}{2}\left(\rho+\rho^{-1}\right)\right)\right|
$$

for each $\rho>\rho_{0}$.

For $n=1$ the corresponding result is slightly different.

THEOREM 6.2. Let $n=1$.

a) For $s=1$, there exists $\rho_{0}$ such that

$$
\max _{z \in \mathcal{E}_{\rho}}\left|K_{1,1}(z)\right|=\left|K_{1,1}\left(\frac{1}{2}\left(\rho+\rho^{-1}\right)\right)\right|,
$$

for each $\rho>\rho_{0}$.

b) For $s>1$, there exists $\rho_{0}=\rho_{0}(s)$ such that

$$
\max _{z \in \mathcal{E}_{\rho}}\left|K_{1, s}(z)\right|=\left|K_{1, s}\left(\frac{i}{2}\left(\rho-\rho^{-1}\right)\right)\right|,
$$

for each $\rho>\rho_{0}$.

In order to derive an $L^{1}$-error bound, we use (1.4) and study the quantity

$$
L_{n, s}\left(\mathcal{E}_{\rho}\right)=\frac{1}{2 \pi} \oint_{\mathcal{E}_{\rho}}\left|K_{n, s}(z)\right||d z|,
$$

where the kernel $K_{n, s}(z)$ is given by (6.5).

Let $z=\frac{1}{2}\left(u+u^{-1}\right)$, where $u=\rho e^{i \theta}$. Letting $a_{j}=\frac{1}{2}\left(\rho^{j}+\rho^{-j}\right)$, for $j \in \mathbb{N}$, we obtain

$$
L_{n, s}\left(\mathcal{E}_{\rho}\right)=\frac{1}{2^{s+\frac{1}{2}}} \int_{0}^{\pi} \frac{\left|\sum_{k=0}^{s}\left(\left(\begin{array}{c}
2 s+1 \\
k-1
\end{array}\right)-\left(\begin{array}{c}
2 s+1 \\
k
\end{array}\right)\right) u^{-2 s n+2 k n-2 n}\right|}{\sqrt{a_{2 n}-\cos 2 n \theta}\left(a_{2 n}+\cos 2 n \theta\right)^{s}} d \theta .
$$

After some computations, we get the following result and consequently the $L^{1}$-error bound.

THEOREM 6.3. For the expression $L_{n, s}\left(\mathcal{E}_{\rho}\right)$ given by (6.6), it holds that

$$
L_{n, s}\left(\mathcal{E}_{\rho}\right) \leqslant \frac{\pi \sqrt{Q_{s}\left(\rho^{4 n}\right)}}{\rho^{n}\left(\rho^{4 n}-1\right)^{2 s}}
$$


where

$$
\begin{aligned}
Q_{s}\left(\rho^{4 n}\right) & =2 \sum_{k=0}^{s}{ }^{\prime}(-1)^{k}\left(\sum_{j=0}^{s-k} D_{s, j} D_{s, j+k} \rho^{4 j n}\right)\left(\sum_{i=0}^{2 s-1} E_{s, i}^{k}\left(\rho^{4 n}-1\right)^{i}\right), \\
D_{s, j} & =\left(\begin{array}{c}
2 s+1 \\
j-1
\end{array}\right)-\left(\begin{array}{c}
2 s+1 \\
j
\end{array}\right), \quad E_{s, i}^{k}=\left(\begin{array}{c}
2 s+k-1 \\
i
\end{array}\right)\left(\begin{array}{c}
4 s-i-2 \\
2 s-1
\end{array}\right) .
\end{aligned}
$$

Let us now derive the error bound based on expanding the error term. If $f$ is an analytic function in the interior of $\mathcal{E}_{\rho}$, it can be expanded as

$$
f(z)=\sum_{k=0}^{\infty} \alpha_{k} T_{k}(z)
$$

where

$$
\alpha_{k}=\frac{1}{\pi} \int_{-1}^{1}\left(1-t^{2}\right)^{-1 / 2} f(t) T_{k}(t) d t .
$$

This series converges for all $z$ in the interior of $\mathcal{E}_{\rho}$. The prime symbol on the summation sign means that the first term of the sum is to be halved.

The expansion of the kernel (6.5) is given by

$$
K_{n, s}(z)=2 \pi \sum_{k=0}^{s} \sum_{i, j=0}^{\infty} C_{s, k}(-1)^{j}\left(\begin{array}{c}
2 s-1+j \\
2 s-1
\end{array}\right)\left(1+\left[\frac{i}{n}\right]\right) u^{p},
$$

where $[x]$ denotes the integer part of a real number $x$,

$$
C_{s, k}=\left(\begin{array}{c}
2 s+1 \\
s-k-1
\end{array}\right)-\left(\begin{array}{c}
2 s+1 \\
s-k
\end{array}\right)
$$

and $p=-(2 s+3) n-2((j+k) n+i)-1$. The coefficient for $u^{-(2 s+3) n-2(a n+b)-1}$ with $0 \leqslant a$ and $0 \leqslant b \leqslant n-1$ in the above expression equals

$$
\omega_{n, 2 a n+2 b}^{(s)}=2 \pi \sum_{k=0}^{s} \sum_{j=0}^{a-k} C_{s, k}(-1)^{j}\left(\begin{array}{c}
2 s-1+j \\
2 s-1
\end{array}\right)(a-k-j+1),
$$

so we obtain

$$
K_{n, s}(z)=\sum_{j=0}^{\infty} \sum_{i=0}^{n-1} \omega_{n, 2 j n+2 i}^{(s)} u^{-(2 s+3) n-2 j n-2 i-1} .
$$

THEOREM 6.4. The remainder term $R_{n, s}\left(f T_{n}\right)$ can be represented in the form

$$
R_{n, s}\left(f T_{n}\right)=\sum_{k=0}^{\infty} \alpha_{(2 s+3) n+k} \varepsilon_{n, k}^{(s)},
$$

where the coefficients $\varepsilon_{n, k}^{(s)}$ are independent of $f$. Furthermore, $\varepsilon_{n, 2 j+1}^{(s)}=0$, for $j \geq 0$, and

$$
\varepsilon_{n, k}^{(s)}= \begin{cases}\frac{\pi}{2} \sum_{l=0}^{s} \sum_{i=0}^{j-l}\left(\left(\begin{array}{c}
2 s+1 \\
s-l-1
\end{array}\right)-\left(\begin{array}{c}
2 s+1 \\
s-l
\end{array}\right)\right)(-1)^{i}\left(\begin{array}{c}
2 s-1+i \\
2 s-1
\end{array}\right), & k=2 j n, \\
0, & k \neq 2 j n,\end{cases}
$$

for $j=0,1, \ldots$ 
Thus, we have

$$
\left|R_{n, s}\left(f T_{n}\right)\right| \leqslant \sum_{k=0}^{\infty}\left|\alpha_{(2 s+3) n+k}\right|\left|\varepsilon_{n, k}^{(s)}\right| .
$$

In general, the Chebyshev coefficients $\alpha_{k}$ in (6.7) are not known. In particular, under our assumptions ([10]),

$$
\left|\alpha_{k}\right| \leqslant \frac{2}{\rho^{k}}\left(\max _{z \in \mathcal{E}_{\rho}}|f(z)|\right)
$$

Now we get

$$
\left|R_{n, s}\left(f T_{n}\right)\right| \leqslant \pi \max _{z \in \mathcal{E}_{\rho}}|f(z)| F(\rho)
$$

where

$$
F(\rho)=\frac{1}{\rho^{(2 s+3) n}} \sum_{j=0}^{\infty}\left|\varepsilon_{n, 2 j n}^{(s)}\right| \rho^{-2 j n}
$$

and $\varepsilon_{n, 2 j n}^{(s)}$ are defined by (6.8). Although the $\varepsilon_{n, 2 j n}^{(s)}$ are sums themselves, it turns out that $F(\rho)$ can be simplified to a single finite sum; see [54] for a proof.

LEMMA 6.1. For $F(\rho)$ given by (6.10) with $\rho>1$, it holds that

$$
F(\rho)=\frac{\sum_{l=0}^{s}(-1)^{l+1}\left(\left(\begin{array}{c}
2 s+1 \\
s-l-1
\end{array}\right)-\left(\begin{array}{c}
2 s+1 \\
s-l
\end{array}\right)\right) \rho^{2(s-l) n}}{\rho^{n}\left(\rho^{2 n}-1\right)^{2 s}\left(\rho^{2 n}+1\right)} .
$$

Now we can formulate the main result.

THEOREM 6.5. For $s \in \mathbb{N}$, the estimate (6.9) can be expressed in the form

$$
\left|R_{n, s}\left(f T_{n}\right)\right| \leqslant \pi \max _{z \in \mathcal{E}_{\rho}}|f(z)| \frac{\sum_{l=0}^{s}(-1)^{l+1}\left(\left(\begin{array}{c}
2 s+1 \\
s-l-1
\end{array}\right)-\left(\begin{array}{c}
2 s+1 \\
s-l
\end{array}\right)\right) \rho^{2(s-l) n}}{\rho^{n}\left(\rho^{2 n}-1\right)^{2 s}\left(\rho^{2 n}+1\right)} .
$$

EXAMPLE 6.1. Let us calculate the integral

$$
\int_{-1}^{1} T_{n}(t) f(t) \frac{d t}{\sqrt{1-t^{2}}}, \quad f(z)=e^{\cos (\omega z)}, \quad \omega>0
$$

by using the quadrature formula (6.2). We denote the $L^{\infty}$-error bounds, the $L^{1}$-error bounds, and the error bounds based on expanding the remainder term in a series by $\left|R_{n, s}^{(i)}(f)\right| \leqslant r_{i}(f)$, for $i=1,2,3$, respectively and calculate them for some values of $n, s$, and $\omega$. The obtained estimates hold for $\mathcal{E}_{\rho}, \rho>1$. We have

$$
\max _{z \in \mathcal{E}_{\rho}}\left|e^{\cos (\omega z)}\right|=e^{\cosh \left(\omega b_{1}\right)}, \quad b_{1}=\frac{1}{2}\left(\rho-\rho^{-1}\right) .
$$

"Error" is the actual (sharp) error and $I_{\omega}$ is the exact value of the integral. The results are reported in Table 6.1. 


\section{ETNA}

Kent State University and Johann Radon Institute (RICAM)

TABLE 6.1

The values of the derived bounds $r_{1}\left(f_{1}\right), r_{2}\left(f_{1}\right), r_{3}\left(f_{1}\right)$, for some values of $n, s, \omega$.

\begin{tabular}{c|r|r|r|r|r}
$n, s, \omega$ & $r_{1}\left(f_{1}\right)$ & $r_{2}\left(f_{1}\right)$ & $r_{3}\left(f_{1}\right)$ & Error & $I_{\omega}$ \\
\hline $8,1,0.5$ & $5.54(-39)$ & $5.50(-39)$ & $5.50(-39)$ & $3.34(-40)$ & $1.08 \ldots(-6)$ \\
$8,2,0.5$ & $1.21(-56)$ & $1.21(-56)$ & $1.21(-56)$ & $6.03(-58)$ & $1.08 \ldots(-6)$ \\
$8,1,5$ & $1.89(-5)$ & $1.16(-5)$ & $1.16(-5)$ & $5.81(-7)$ & $3.11 \ldots(-1)$ \\
$8,2,5$ & $8.23(-9)$ & $5.39(-9)$ & $5.39(-9)$ & $2.25(-10)$ & $3.11 \ldots(-1)$ \\
$8,1,10$ & $3.14(-1)$ & $9.48(-2)$ & $9.65(-2)$ & $4.06(-3)$ & $1.07 \ldots(+0)$ \\
$8,2,10$ & $1.93(-2)$ & $6.80(-3)$ & $7.10(-3)$ & $1.98(-4)$ & $1.07 \ldots(+0)$ \\
$10,1,0.5$ & $3.30(-50)$ & $3.29(-50)$ & $3.29(-50)$ & $1.75(-51)$ & $-1.29 \ldots(-8)$ \\
$10,2,0.5$ & $8.16(-73)$ & $8.12(-73)$ & $8.12(-73)$ & $3.56(-74)$ & $-1.29 \ldots(-8)$ \\
$10,1,5$ & $9.30(-8)$ & $5.96(-8)$ & $5.96(-8)$ & $2.65(-9)$ & $-1.72 \ldots(-1)$ \\
$10,2,5$ & $2.35(-12)$ & $1.60(-12)$ & $1.60(-12)$ & $5.93(-14)$ & $-1.72 \ldots(-1)$ \\
$10,1,10$ & $3.36(-2)$ & $1.09(-2)$ & $1.09(-2)$ & $3.95(-4)$ & $-8.80 \ldots(-1)$ \\
$10,2,10$ & $5.72(-4)$ & $2.09(-4)$ & $2.12(-4)$ & $6.01(-6)$ & $-8.80 \ldots(-1)$ \\
$14,1,0.5$ & $3.26(-73)$ & $3.25(-73)$ & $3.25(-73)$ & $1.42(-74)$ & $-1.37 \ldots(-12)$ \\
$14,2,0.5$ & $6.75(-106)$ & $6.73(-106)$ & $6.73(-106)$ & $2.43(-107)$ & $-1.37 \ldots(-12)$ \\
$14,1,5$ & $9.42(-13)$ & $6.38(-13)$ & $6.38(-13)$ & $2.37(-14)$ & $-4.23 \ldots(-2)$ \\
$14,2,5$ & $5.79(-20)$ & $4.10(-20)$ & $4.10(-20)$ & $2.72(-21)$ & $-4.23 \ldots(-2)$ \\
$14,1,10$ & $2.32(-4)$ & $8.36(-5)$ & $8.36(-5)$ & $2.45(-6)$ & $9.03 \ldots(-2)$ \\
$14,2,10$ & $2.39(-7)$ & $9.50(-8)$ & $9.51(-8)$ & $2.30(-9)$ & $9.03 \ldots(-2)$
\end{tabular}

7. Error bounds for Gaussian-type quadrature formulas on circles. In this section we describe, briefly, effective error estimates for quadrature formulas for analytic functions on concentric circles, as this is related to an approach used by Gautschi and Varga [21]. The methods described here for Gauss-type quadrature formulas also are applied to Gauss-Lobatto, Gauss-Radau, Gauss-Kronrod, and Fejér-type quadratures. In certain spaces of analytic functions, the error (remainder) term of a quadrature formula is a bounded linear functional. We mention here the methods used to compute explicitly or in some cases to estimate the norm of the error functional. For a detailed survey; see Notaris [63].

We consider the Gaussian quadrature formula (1.9) by taking $\Gamma$ to be $\mathcal{C}_{r}$ instead of $\mathcal{E}_{\rho}$, where

$$
\mathcal{C}_{r}=\{z \in \mathbb{C}|| z \mid=r\}, \quad r>1
$$

We have that

$$
R_{n}(f)=\frac{1}{2 \pi i} \oint_{\mathcal{C}_{r}} K_{n}(z) f(z) d z
$$

where

$$
K_{n}(z)=R_{n}\left(\frac{1}{z-\cdot}\right)
$$

and

$$
\left|R_{n}(f)\right| \leq \frac{l\left(\mathcal{C}_{r}\right)}{2 \pi}\left(\max _{z \in \mathcal{C}_{r}}\left|K_{n}(z)\right|\right)\left(\max _{z \in \mathcal{C}_{r}}|f(z)|\right)
$$

where $l\left(\mathcal{C}_{r}\right)=2 \pi r$. 
If $f$ is an analytic function in the interior of $\mathcal{C}_{r}$, then it can be written as

$$
f(z)=\sum_{k=0}^{\infty} a_{k} z^{k}, \quad z \in \mathcal{C}_{r} .
$$

Define

$$
X_{r}=\left\{f \mid f \text { analytic in } \mathcal{C}_{r} \text { and }|f|_{r}<\infty\right\}
$$

where

$$
|f|_{r}=\sup \left\{\left|a_{k}\right| r^{k} \mid k \in \mathbb{N}_{0} \text { and } R_{n}\left(t^{k}\right) \neq 0\right\}
$$

is a seminorm in $X_{r}$. It can be shown that $R_{n}$ is a continuous linear functional in $\left(X_{r},|\cdot|_{r}\right)$, and its norm is given by (see [2, 4, 26])

$$
\left\|R_{n}\right\|=\sum_{k=0}^{\infty} \frac{\left|R_{n}\left(t^{k}\right)\right|}{r^{k}} .
$$

Under the additional hypotheses that

$$
\frac{w(\cdot)}{w(-\cdot)} \text { is nondecreasing on }(-1,1)
$$

or

$$
\frac{w(\cdot)}{w(-\cdot)} \text { is nonincreasing on }(-1,1)
$$

we can derive the following representations:

$$
\left\|R_{n}\right\|=\frac{r}{\pi_{n}(r)} \int_{-1}^{1} \frac{\pi_{n}(t)}{r-t} w(t) d t
$$

if $w$ satisfies (7.4) and

$$
\left\|R_{n}\right\|=\frac{r}{\pi_{n}(-r)} \int_{-1}^{1} \frac{\pi_{n}(t)}{r+t} w(t) d t
$$

if $w$ satisfies (7.5) (see [3]).

For some classes of weight functions $w(t)$, the integrals in (7.6) and (7.7) can be computed explicitly or in some cases estimated. Therefore if $f \in X_{R}$, then for every $r \in(1, R]$

$$
\left|R_{n}(f)\right| \leq\left\|R_{n}\right\||f|_{r}
$$

and consequently

$$
\left|R_{n}(f)\right| \leq \inf _{1<r \leq R}\left(\left\|R_{n}\right\||f|_{r}\right) .
$$

The norm $\left\|R_{n}\right\|$ has been computed explicitly in [2,4] for the Chebyshev weight functions of any of the four kinds. For example, for the Jacobi weight function $w(t)=(1-t)^{\alpha}(1+$ $t)^{\beta}, \alpha, \beta>-1$, one has

$$
\frac{w(t)}{w(-t)}=\left(\frac{1+t}{1-t}\right)^{\beta-\alpha}
$$




\section{ETNA}

Kent State University and

Johann Radon Institute (RICAM)

which is increasing on $(-1,1)$ if $\alpha<\beta$ and decreasing if $\alpha>\beta$. Therefore, we have (7.4) if $\alpha \leq \beta$ and (7.5) otherwise.

Gautschi and Varga in [21, Theorem 3.1] showed that if $\Gamma=\mathcal{C}_{r}$ and $w$ satisfies either (7.4) or (7.5), then

$$
\max _{|z|=r}\left|K_{n}(z)\right|=\left\{\begin{array}{ll}
K_{n}(r) & \text { if } w \text { satisfies (7.4) } \\
\left|K_{n}(-r)\right| & \text { if } w \text { satisfies (7.5) }
\end{array}=\sum_{k=0}^{\infty} \frac{\left|R_{n}\left(t^{k}\right)\right|}{r^{k+1}},\right.
$$

that is, by (7.3),

$$
\max _{|z|=r}\left|K_{n}(z)\right|=\frac{\left\|R_{n}\right\|}{r} .
$$

Equation (7.1) gives

$$
\left|R_{n}(f)\right| \leq\left\|R_{n}\right\| \max _{|z|=r}|f(z)| .
$$

In particular, if $f$ is analytic in the interior of $\mathcal{C}_{R}$, then

$$
\left|R_{n}(f)\right| \leq \inf _{1<r \leq R}\left(\left\|R_{n}\right\| \max _{|z|=r}|f(z)|\right) .
$$

The papers [57, 58, 59, 61, 62, 63, 65, 67] by S. Notaris focus on this subject, with [63] being a review paper. The paper [60] deals with some integral formulas for Chebyshev polynomials with an application to the error term of interpolatory formulas for analytic functions (Sections 3 and 4). The paper [64] deals with some product formulas for Chebyshev weight functions with Chebyshev abscissae, and Section 3.2 in that paper studies the error term of these formulas for analytic functions. Similarly, the paper [66] deals with a corrected Fejér quadrature formula of the second kind, and Section 3.2 in that paper studies the error term of this corrected formula for analytic functions.

Peherstorfer [72] gave an explicit expression for the kernel of the error functional for Gaussian quadrature formulas with respect to weight functions of Bernstein-Szegő type of the form $(1-t)^{\alpha}(1+t)^{\beta} / \rho(t), t \in(-1,1)$, where $\alpha, \beta \in\{-1 / 2,1 / 2\}$ and $\rho$ is a polynomial of arbitrary degree which is positive on $[-1,1]$. Using this result, the norm can easily be calculated explicitly for a wide subclass of these weight functions. Our two paper on the subject, in which we use circles, are [41, 46].

Acknowledgments. We are indebted to a referee and Lothar Reichel for making suggestions that have improved the paper.

\section{REFERENCES}

[1] I. N. ACHIESER, Theory of Approximation, Dover, New York, 1992.

[2] G. AKRIVIS, Fehlerabschätzungen für Gauss-Quadraturformeln, Numer. Math., 44 (1984), pp. 261-278.

[3] — The error norm of certain Gaussian quadrature formulae, Math. Comp., 45 (1985), pp. 513-519.

[4] G. AKRIVIS AND A. BURGSTALLER, Fehlerabschätzungen für nichtsymmetrische Gauss-Quadraturformeln, Numer. Math., 47 (1985), pp. 535-543.

[5] S. BERnStEIN, Sur les polynomes orthogonaux relatifs à un segment fini, J. Math. Pures Appl., 9 (1930), pp. 127-177.

[6] B. Bojanov And G. Petrova, Quadrature formulas for Fourier coefficients, J. Comput. Appl. Math., 231 (2009), pp. 378-391.

[7] B. DE LA CALLE YSERN, Error bounds for rational quadrature formulas of analytic functions, Numer. Math., 101 (2005), pp. 251-271. 
[8] B. DE La CALle YSERn AND P. GonZÁlez-Vera, Rational quadrature formulas on the unit circle with arbitrary poles, Numer. Math., 107 (2007), pp. 259-287.

[9] D. LJ. DJukić, A. V. PejČEv, AND M. M. SPAleviĆ, The error bounds of Gauss-Kronrod quadrature formulas with Bernstein-Szegö weight functions, Numer. Algorithms, 77 (2018), pp. 1003-1028.

[10] D. ElLiotT, The evaluation and estimation of the coefficients in the Chebyshev series expansion of a functions, Math. Comp., 18 (1964), pp. 82-90.

[11] W. GAUTSCHI, On Padé approximants associated with Hamburger series, Calcolo, 20 (1983), pp. 111-127.

[12] - On the remainder term for analytic functions of Gauss-Lobatto and Gauss-Radau quadratures, Rocky Mountain J. Math., 21 (1991), pp. 209-226.

[13] - Orthogonal Polynomials: Computation and Approximation, Oxford University Press, Oxford, 2004.

[14] — OPQ suite, Software, 2001. http://www. cs . purdue.edu/archives/2001/wxg/codes

[15] _ High-precision Gauss-Turán quadrature rules for Laguerre and Hermite weight functions, Numer. Algorithms, 67 (2014), pp. 59-72.

[16] W. GAUTSCHI AND S. LI, The remainder term for analytic functions of Gauss-Radau and Gauss-Lobatto quadrature rules with multiple end points, J. Comput. Appl. Math., 33 (1990), pp. 315-329.

[17] _ A set of orthogonal polynomials induced by a given orthogonal polynomial, Aequationes Math., 46 (1993), pp. 174-198.

[18] W. GaUtsChI AND G. V. Milovanović, S-orthogonality and construction of Gauss-Turán-type quadrature formulas, J. Comput. Appl. Math., 86 (1997), pp. 205-218.

[19] W. GAUTSCHI AND S. E. NOTARIS, Gauss-Kronrod quadrature formulas for weight function of BernsteinSzegö type, J. Comput. Appl. Math., 25 (1989), pp. 199-224; erratum in J. Comput. Appl. Math., 27 (1989), pp. 429.

[20] W. GAuTSCHI, E. Tychopoulos, AND R. S. VARGA, A note on the contour integral representation of the remainder term for a Gauss-Chebyshev quadrature rule, SIAM J. Numer. Anal., 27 (1990), pp. 219-224.

[21] W. GAUTSCHI AND R. S. VARGA, Error bounds for Gaussian quadrature of analytic functions, SIAM J. Numer. Anal., 20 (1983), pp. 1170-1186.

[22] A. GhizZetTi And A. Ossicini, Quadrature Formulas, Akademie-Verlag, Berlin, 1970.

[23] V. L. GonČAROV, Theory of Interpolation and Approximation of Functions, GITTL, Moscow, 1954.

[24] L. GORI AND C. A. MICCHELLI, On weight functions which admit explicit Gauss-Turán quadrature formulas, Math. Comp., 69 (1996), pp. 1567-1581.

[25] I. S. GRAdShteYn AND I. M. RyzhiK, Tables of Integrals, Series and Products, 6th ed., Academic Press, San Diego, 2000.

[26] G. HÄMMERLIN, Fehlerabschätzungen bei numerischer Integration nach Gauss. In Methoden und Verfahren der Mathematischen Physik vol. 6, B. Brosowski and E. Martensen, eds., Biblographisches Institut, Mannheim, 1972, pp. 153-163.

[27] D. B. HUNTER, Some properties of orthogonal polynomials, Math. Comp., 29 (1975), pp. 559-565.

[28] - Some error expansions for Gaussian quadrature, BIT, 35 (1995), pp. 64-82.

[29] D. B. HunTER AND G. NiKOLOv, On the error term of symmetric Gauss-Lobatto quadrature formulas for analytic functions, Math. Comp., 69 (2000), pp. 269-282.

[30] D. P. LAURIE, Anti-Gaussian quadrature formulas, Math. Comp., 65 (1996), pp. 739-747.

[31] C. A. Micchelli AND T. J. Rivlin, Turán formulas and highest precision quadrature rules for Chebyshev coefficients, IBM J. Res. Develop., 16 (1972), pp. 372-379.

[32] LJ. V. MIHIĆ, The remainder term of Gauss-Radau quadrature rule with single and double end point, Publ. Inst. Math. Beograd (N.S.), 102 (2017), pp. 73-83.

[33] LJ. V. Minić, A. V. PEJČEV, AND M. M. SPALEVIĆ, Error bounds for Gauss-Lobatto quadrature formula with multiple end points with Chebyshev weight function of the third and the fourth kind, Filomat, 30 (2016), pp. 231-239.

[34] — Error estimations of Turán formulas with Gori-Micchelli and generalized Chebyshev weight functions, Filomat, 32 (2018), pp. 6927-6936.

[35] G. V. MiLovanović, Quadratures with multiple nodes, power orthogonality, and moment-preserving spline approximation, J. Comput. Appl. Math., 127 (2001), pp. 267-286.

[36] G. V. Milovanović, R. ORIVE, AND M. M. Spalević, Quadrature with multiple nodes for FourierChebyshev coefficients, IMA J. Numer. Anal., 39 (2019), pp. 271-296.

[37] G. V. Milovanović, A. V. PejČEv, AND M. M. Spalević, A note on an error bound of Gauss-Turán quadrature with the Chebyshev weight function of the first kind, Filomat, 27 (2013), pp. 1037-1042.

[38] G. V. Milovanović, M. S. Pranić, AND M. M. Spalević, Quadrature with multiple nodes, power orthogonality, and moment-preserving spline approximation, part II, Appl. Anal. Discrete Math., 13 (2019), pp. 1-27.

[39] G. V. Milovanović AND M. M. Spalević, Error bounds for Gauss-Turán quadrature formulas of analytic functions, Math. Comp., 72 (2003), pp. 1855-1872.

[40] — Error analysis in some Gauss-Turán-Radau and Gauss-Turán-Lobatto quadratures for analytic functions, J. Comput. Appl. Math., 164-165 (2004), pp. 569-586. 


\section{ETNA}

Kent State University and

Johann Radon Institute (RICAM)

[41] _ Bounds of the error of Gauss-Turán-type quadratures, J. Comput. Appl. Math., 178 (2005), pp. 333346.

[42] - An error expansion for some Gauss-Turán quadratures and $L^{1}$-estimates of the remainder term, BIT, 45 (2005), pp. 117-136.

[43] — Gauss-Turán quadratures of Kronrod Type for generalized Chebyshev weight functions, Calcolo, 43 (2006), pp. 171-195.

[44] - Quadrature rules with multiple nodes for evaluating integrals with strong singularities, J. Comput. Appl. Math., 189 (2006), pp. 689-702.

[45] - A note on the bounds of the error of Gauss-Turán-type quadratures, J. Comput. Appl. Math., 200 (2007), pp. 276-282.

[46] - Monotonicity of the error in Gauss-Turán quadratures for analytic functions, ANZIAM J., 48 (2007), pp. 567-581.

[47] - Kronrod extensions with multiple nodes of quadrature formulas for Fourier coefficients, Math. Comp., 83 (2014), pp. 1207-1231.

[48] G. V. Milovanović, M. M. Spalević, And A. S. CvetKović, Calculation of Gaussian type quadratures with multiple nodes, Math. Comput. Modelling, 39 (2004), pp. 325-347.

[49] G. V. Milovanović, M. M. Spalević, And M. S. PRanić, Maximum of the modulus of kernels in Gauss-Turán quadratures, Math. Comp., 77 (2008), pp. 985-994.

[50] - On the remainder term of Gauss-Radau quadratures for analytic functions, J. Comput. Appl. Math., 218 (2008), pp. 281-289.

[51] - Error estimates for Gaussian quadratures of analytic functions, J. Comput. Appl. Math., 233 (2009), pp. 802-807.

[52] - Error estimates for Gauss-Turán quadratures and their Kronrod extensions, IMA J. Numer. Anal., 29 (2009), pp. 486-507.

[53] - Bounds of the error of Gauss-Turán-type quadratures, II, Appl. Numer. Math., 60 (2010), pp. 1-9.

[54] R. M. MutavdžIĆ, A. V. PejČEV, And M. M. SPALEVIĆ, Error bounds for Kronrod extension of generalizations of Micchelli-Rivlin quadrature formula for analytic functions, Electron. Trans. Numer. Anal., 50 (2018), pp. 20-35.

http://etna.ricam.oeaw.ac.at/vol.50.2018/pp20-35.dir/pp20-35.pdf

[55] - The error bounds of Gauss-Lobatto quadrature for weight functions of Bernstein-Szegó type, Appl. Anal. Discrete Math., 13 (2019), pp. 733-745.

[56] I. P. MYsovsKiH, Lectures of Numerical Methods, Wolters-Noordhoff Publishing, Groningen, 1969.

[57] S. E. NOTARIS, The error norm of Gaussian quadrature formulas for weight functions of Bernstein-Szegö type, Numer. Math., 57 (1990), pp. 271-283.

[58] - Error bounds for Gauss-Kronrod quadrature formulas of analytic functions, Numer. Math., 64 (1993), pp. 371-380.

[59] - The error norm of Gauss-Lobatto quadrature formulas for weight functions of Bernstein-Szegö type, Numer. Math., 64 (1993), pp. 381-393.

[60] - Integral formulas for Chebyshev polynomials and the error term of interpolatory quadrature formulas for analytic functions, Math. Comp., 75 (2006), pp. 1217-1231.

[61] - The error norm of Gauss-Kronrod quadrature formulas for weight functions of Bernstein-Szegö type, Numer. Math., 103 (2006), pp. 99-127.

[62] - The error norm of Gauss-Radau quadrature formulas for Chebyshev weight functions, BIT, 50 (2010), pp. 123-147.

[63] - The error norm of quadrature formulas, Numer. Algorithms, 60 (2012), pp. 555-578.

[64] - Product integration rules for Chebyshev weight functions with Chebyshev abscissae, J. Comput. Appl. Math., 257 (2014), pp. 180-194.

[65] - The error norm of Gauss-Radau quadrature formulas for Bernstein-Szegö weight functions, Math. Comp., 84 (2015), pp. 2843-2865.

[66] - On a corrected Fejér quadrature formula of the second kind, Numer. Math., 133 (2016), pp. 279-302.

[67] - The error norm of Clenshaw-Curtis and related quadrature formulas, BIT, 56 (2016), pp. 705-728.

[68] _ Gauss-Kronrod quadrature formulas - A survey of fifty years of research, Electron. Trans. Numer. Anal., 45 (2016), pp. 371-404. http://etna.ricam.oeaw.ac.at/vol.45.2016/pp371-404.dir/pp371-404.pdf

[69] R. ORIVE, A. V. PEJČEV, AND M. M. Spalević, The error bounds of Gauss quadrature formula for the modified weight functions of Chebyshev type, Appl. Math. Comput., 369 (2020), Art. 124806, 22 pages.

[70] A. OssicINI AND F. Ros ATI, Funzioni caratteristiche nelle formule di quadratura gaussiane con nodi multipli, Boll. Un. Mat. Ital., 11 (1975), pp. 224-237.

[71] - Sulla convrgenza dei funzionali ipergaussiani, Rend. Mat., 11 (1978), pp. 97-108.

[72] F. PEHERSTORFER, On the remainder of Gaussian quadrature formulas for Bernstein-Szegó weight functions, Math. Comp., 60 (1993). pp. 317-325. 
[73] A. V. PEJČEV, Error bounds for Gauss-type quadratures with Bernstein-Szegö weights, Numer. Algorithms, 66 (2014), pp. 569-590.

[74] _ Error estimates of Gaussian quadrature formulas with the third class of Bernstein-Szegó weights, Appl. Anal. Discrete Math., 11 (2017), pp. 451-469.

[75] A. V. PEJČEV AND LJ. V. MinIĆ, Errors of Gauss-Radau and Gauss-Lobatto quadratures with double end point, Appl. Anal. Discrete Math., 13 (2019), pp. 463-477.

[76] A. V. PEJČEV AND M. M. SPALEVIĆ, On the remainder term of Gauss-Radau quadrature with Chebyshev weight of the third kind for analytic functions, Appl. Math. Comput., 219 (2012), pp. 2760-2765.

[77] _ _ Error bounds for Gaussian quadrature formulas with Bernstein-Szegó weights that are rational modifications of Chebyshev weight functions of the second kind, IMA J. Numer. Anal., 32 (2012), pp. 1733-1754.

[78] - Error bounds of Micchelli-Rivlin quadrature formula for analytic functions, J. Approx. Theory, 169 (2013), pp. 23-34.

[79] - Error bounds of the Micchelli-Sharma quadrature formula for analytic functions, J. Comput. Appl. Math., 259 (2014), pp. 48-56.

[80] - The error bounds of Gauss-Radau quadrature formulas with Bernstein-Szegó weight functions, Numer. Math., 133 (2016), pp. 177-201.

[81] _ Error bounds of a quadrature formula with multiple nodes for the Fourier-Chebyshev coefficients for analytic function, Sci. China Math., 62 (2019), pp. 1657-1668.

[82] R. SCHERER AND T. SCHIRA, Estimating quadrature errors for analytic functions using kernel representations and biorthogonal systems, Numer. Math., 84 (2000), pp. 497-518.

[83] T. SCHIRA, The remainder term for analytic functions of Gauss-Lobatto quadratures, J. Comput. Appl. Math., 76 (1996), pp. 171-193.

[84] _ The remainder term for analytic functions of symmetric Gaussian quadratures, Math. Comp., 66 (1997), pp. 297-310.

[85] Y. G. SHI AND G. XU, Construction of $\sigma$-orthogonal polynomials and Gaussian quadrature formulas, Adv. Comput. Math., 27 (2007), pp. 79-94.

[86] M. M. SPAlEvić, Error estimates of anti-Gaussian quadrature formulas, J. Comput. Appl. Math., 236 (2012), pp. 3542-3555.

[87] _ Error bounds of Gaussian quadrature formulas for one class of Bernstein-Szegö weights, Math. Comp., 82 (2013), pp. 1037-1056.

[88] _ _ Error bounds of Gaussian quadratures for one class of Bernstein-Szegó weight functions, in The Third Mathematical Conference of Republic of Srpska, Trebinje, June 7 and 8, 2013, pp. 161-176.

[89] _ Error bounds and estimates for Gauss-Turán quadrature formulas of analytic functions, SIAM J. Numer. Anal., 52 (2014), pp. 443-467.

[90] M. M. SPAleviĆ AND M. S. PRANIĆ, Error bounds of certain Gaussian quadrature formulas, J. Comput. Appl. Math., 234 (2010), pp. 1049-1057.

[91] _ The remainder term of Gauss-Turán quadratures for analytic functions, in Approximation and Computations, W. Gautschi, G. Mastroianni, and T. M. Rassias, eds., Springer Optimization and Its Applications 42, Springer, New York, 2011, pp. 253-266.

[92] M. M. SPAlević, M. S. Pranić, AND A. V. PejČEv, Maximum of the modulus of kernels of Gaussian quadrature formulas for one class of Bernstein-Szegö weight functions, Appl. Math. Comput., 218 (2012), pp. 5746-5756.

[93] F. STENGER, Bounds on the error of Gauss-type quadratures, Numer. Math., 8 (1966), pp. 150-160.

[94] P. TURÁN, On the theory of the mechanical quadrature, Acta Sci. Math. (Szeged), 12 (1950), pp. 30-37.

[95] B. VON SYDOw, Error estimates for Gaussian quadrature formulas, Numer. Math., 29 (1977), pp. 59-64.

[96] C. ZHOU, Convergence of Gaussian quadrature formulas on infinite intervals, J. Approx. Theory, 123 (2003), pp. 280-294. 